\title{
RINGS OF DIFFERENTIAL OPERATORS ON INVARIANT RINGS OF TORI
}

\author{
IAN M. MUSSON
}

\begin{abstract}
Let $k$ be an algebraically closed field of characteristic zero and $G$ a torus acting diagonally on $k^{s}$. For a subset $\beta$ of $\mathbf{s}=\{1,2, \ldots, s\}$, set $U_{\beta}=\left\{u \in k^{s} \mid u_{j} \neq 0\right.$ if $j \in \beta\}$. Then $G$ acts on $\mathcal{O}\left(U_{\beta}\right)$, the ring of regular functions on $U_{\beta}$, and we study the ring $D\left(\mathcal{O}\left(U_{\beta}\right)^{G}\right)$ of all differential operators on the invariant ring.

More generally suppose that $\Delta$ is a set of subsets of $\mathbf{s}$, such that each invariant ring $\mathcal{O}\left(U_{\beta}\right)^{G}, \beta \in \Delta$, has the same quotient field. We prove that $\bigcap_{\beta \in \Delta} D\left(\mathcal{O}\left(U_{\beta}\right)^{G}\right)$ is Noetherian and finitely generated as a $k$-algebra.

Now $G$ acts on each $D\left(\mathcal{O}\left(U_{\beta}\right)\right)$ and there is a natural map
\end{abstract}

$$
\theta: \bigcap_{\beta \in د} D\left(\mathcal{O}\left(U_{\beta}\right)\right)^{G i} \rightarrow \bigcap_{\beta \in J} D\left(\mathcal{O}\left(U_{\beta}\right)^{G}\right)=D\left(Y_{د} / G\right)
$$

obtained by restriction of the differential operators. We find necessary and sufficient conditions for $\theta$ to be surjective and describe the kernel of $\theta$.

The algebras $\bigcap_{\beta \in \Delta} D\left(\mathcal{O}\left(U_{\beta}\right)\right)^{G}$ and $\bigcap_{\beta \in\lrcorner} D\left(\mathcal{O}\left(U_{\beta}\right)^{G}\right)$ carry a natural filtration given by the order of the differential operators. We show that the associated graded rings are finitely generated commutative algebras and are Gorensetin rings. We also determine the centers of $\bigcap_{\beta \in \Delta} D\left(\mathcal{O}\left(U_{\beta}\right)\right)^{G}$ and $\bigcap_{\beta \in \Delta} D\left(\mathcal{O}\left(U_{\beta}\right)^{G}\right)$.

Introduction. Throughout this paper $k$ will be an algebraically closed field of characteristic zero. If $K$ is a commutative $k$-algebra we denote by $D_{0}(K)$ the set of $k$-linear maps $K \rightarrow K$ and if $p \geqslant 1$ a $k$-linear map $f: K \rightarrow K$ belongs to $D_{p}(K)$ provided the map $[f, r]$ defined by $[f, r](s)=f(r s)-r f(s)$ for $s \in K$ belongs to $D_{p-1}(K)$ for all $r \in K$. The set $D(K)=\bigcup_{p \geqslant 0} D_{p}(K)$ forms a subring of $\operatorname{End}_{k}(K)$ called the ring of differential operators on $K$. We consider $K$ as a left $D(K)$-module where $f \cdot r=f(r)$ for all $f \in D(K), r \in K$.

The ring of differential operators on the invariant ring of a finite group acting on a polynomial ring has been studied in [Ka, L1 and L2]. We carry out a similar study for the invariant ring of a torus.

We assume that the torus $G$ acts on $k^{s}$ as a group of diagonal matrices. If $\beta$ is a subset of $\mathbf{s}=\{1,2, \ldots, s\}$, then $G$ acts on $U_{\beta}=\left\{u \in k^{s} \mid u_{j} \neq 0\right.$ if $\left.j \in \beta\right\}$. Also $G$ acts on $\mathcal{O}\left(U_{\beta}\right)$ via $(g f)(u)=f\left(g^{-1} u\right)$ for $g \in G, f \in \mathcal{O}\left(U_{\beta}\right), u \in U_{\beta}$. More generally suppose that for some set of subsets $\Delta$ of s we have $Y=\cup_{\beta \in \Delta} U_{\beta}$, where each invariant ring $\mathcal{O}\left(U_{\beta}\right)^{G}$ has the same quotient field $F$. Then each $D\left(\mathcal{O}\left(U_{\beta}\right)^{G}\right)$ is a

Received by the editors June 18, 1986 and, in revised form, December 11, 1986.

1980 Mathematics Subject Classification (1985 Revision). Primary 16A33; Secondary 13N05, 14 L30. 
subring of $D(F)$ and we define

$$
D\left(Y_{\Delta} / G\right)=\bigcap_{\beta \in \Delta} D\left(\mathcal{O}\left(U_{\beta}\right)^{G}\right)
$$

THEOREM A. With the above notation $D\left(Y_{\Delta} / G\right)$ is Noetherian and finitely generated as a k-algebra.

If $\Delta$ consists of a single subset $\Delta=\{\beta\}$ then $D\left(Y_{\Delta} / G\right)=D\left(\mathcal{O}\left(U_{\beta}\right)^{G}\right)$. However the ring $\mathcal{O}\left(U_{\beta}\right)^{G}$ does not in general reflect the geometry of the orbit space $U_{\beta} / G$ very well. A simple example of this is where $G=\mathrm{GL}(1)$ acts on $U_{\phi}=k^{s}$ by scalar multiplication. Then the origin $O$ is the only closed orbit, and $\left(U_{\phi}-O\right) / G \cong \mathbf{P}^{s-1}$ but $\mathcal{O}\left(U_{\phi}\right)^{G}=k$.

However, there are many nonconstant global differential operators on $\mathbf{P}^{s-1}$; see [BB, Example 3.10a]. The definition of $D\left(Y_{\Delta} / G\right)$ given above is designed to accommodate such examples.

We shall write $D\left(U_{\beta}\right)$ and $D(Y)$ in place of $D\left(\mathcal{O}\left(U_{\beta}\right)\right)$ and $D(\mathcal{O}(Y))$.

The group $G$ acts on $D\left(U_{\beta}\right)$ via $(g \cdot d)(f)=g\left(d\left(g^{-1} f\right)\right)$ for $f \in \mathcal{O}\left(U_{\beta}\right), d \in$ $D\left(U_{\beta}\right)$, and $g \in G$. Moreover there is a natural map $\theta_{\beta}: D\left(U_{\beta}\right)^{G} \rightarrow D\left(\mathcal{O}\left(U_{\beta}\right)^{G}\right)$ which is obtained by restriction of the differential operators. Taking intersections over $\beta \in \Delta$, we obtain a map

$$
\theta: D(Y)^{G}=\bigcap_{\beta \in \Delta} D\left(U_{\beta}\right)^{G} \rightarrow \bigcap_{\beta \in \Delta} D\left(\mathcal{O}\left(U_{\beta}\right)^{G}\right)=D\left(Y_{\Delta} / G\right) .
$$

In Theorem $\mathrm{B}$ below we obtain necessary and sufficient conditions for $\theta$ to be surjective and describe the kernel of $\theta$. The corresponding result for the action of a finite group on a polynomial ring is proved in [Ka, Chapter III, Théorème 4 and $\mathbf{L 1}$, Théorème 5].

The statement of Theorem B is given at the beginning of $\S 2$. It is formulated in terms of certain convex rational polyhedral cones which are related to the invariant rings of a torus. For other results on invariant rings of tori which use this approach see [Ho and Ke].

This paper is organized as follows. In $\S 1$ we assemble the results we need on rings of invariants of tori acting on $\mathcal{O}\left(U_{\beta}\right)$ and convex cones. Theorem $\mathrm{B}$ is proved in $\S 2$. In $\S 3$ we describe the centers of $D(Y)^{G}$ and $D\left(Y_{\Delta} / G\right)$ (see Theorem $\mathrm{C}$ and Corollary 3.4) and in Corollary 3.6, obtain an important special case of Theorem B. In $\$ 4$ we filter the rings $D(Y)^{G}$ and $D\left(Y_{\Delta} / G\right)$ by the order of the differential operators, and show that the associated graded rings are finitely generated commutative algebras. Theorem A follows easily from this. We also show that these graded rings are Gorenstein rings (Theorem $\mathrm{D}$ ). This allows us to deduce homological information about modules over $D(Y)^{G}$ and $D\left(Y_{\Delta} / G\right)$; see [L2, Théorème 4.4].

In our companion paper [M] we give some examples and indicate some applications to enveloping algebras of some semisimple Lie algebras.

Index of notation. $\mathbf{Z}_{+}, \mathbf{Q}_{+}$refer to the set of nonnegative integers and rational numbers, respectively. 
Other symbols used frequently in the text are given below in order of appearance. Introduction. $D_{p}(K), D(K), \mathbf{s}, U_{\beta}, \Delta, Y=\cup_{\beta \in \Delta} U_{\beta}, D(Y)^{G}, D\left(Y_{\Delta} / G\right), \theta_{\beta}, \theta$.

1.1. $H_{f}, H_{f}^{0}$

1.3. $\mathbf{Q} \Lambda, \phi, \psi, \psi^{*}, x_{i}, f_{i}$

1.4. $T_{i}, m_{i}, \Lambda_{\beta}, V, H_{i}, \Gamma, M, N$

$\overline{2.0} . \mathscr{H}_{i}, \alpha, J$

2.2. $Q_{i}, P_{i}, A, I(\lambda)$

2.3. $A_{\mathrm{Q}}, \Omega(\lambda)$

2.4. $\Sigma$

2.5. $\Lambda_{b}, \overline{\Omega(\lambda)}, \overline{\Omega_{\beta, i}(\lambda)}, S_{\lambda, \beta, i}, S_{\lambda}$

2.6. $t_{i}, q_{i}, p_{i}, B, B_{\mathbf{Q}}, \omega_{\beta}(\lambda), \omega_{\beta, i}(\lambda)$

2.8. $I$

3.1. $Z(R), Q(R), G K(R)$

3.5. $I_{l}$

4.1. $\mathscr{E}_{t}, \mathscr{D}_{t}, \operatorname{Gr}(R), E_{t}, D_{t}, \Pi_{1}, \Pi_{2}$

4.9. $\mathrm{Kd}(M), d(M), \operatorname{grade}(M)$

4.10. $\operatorname{tr} \operatorname{deg}_{k}(R)$

1. Convex cones and rings of invariants of tori.

1.1. If $f \in\left(\mathbf{Q}^{n}\right)^{*}$ a subset of the form $H_{f}=\left\{\lambda \in \mathbf{Q}^{n} \mid f(\lambda) \geqslant 0\right\}$ is called a halfspace in $\mathbf{Q}^{n}$. We sometimes write $H_{f}^{0}$ for $\operatorname{ker} f$. Given a finite set $S=\left\{v_{1}, \ldots, v_{s}\right\}$ in $\mathbf{Q}^{n}$, the convex rational polyhedral cone, $\operatorname{cone}(S)$ spanned by $S$ is the set

$$
\operatorname{cone}(S)=\sum_{i=1}^{s} \mathbf{Q}^{+} v_{i} \text {. }
$$

THEOREM. (a) If $S$ is a finite subset of $\mathbf{Q}^{n}$, then $\operatorname{cone}(S)$ is a finite intersection of halfspaces. Moreover, if $S$ spans $\mathbf{Q}^{n}$, then $\operatorname{cone}(S)=\bigcap_{i \in I} H_{i}$ where the halfspaces $H_{i}, i \in I$, are determined as those halfspaces $H$ such that $H^{0}$ contains $n-1$ linearly independent elements of $S$.

(b) Conversely any finite intersection of halfspaces in $\mathbf{Q}^{n}$ has the form $\operatorname{cone}(S)$ for some finite set $S$.

Proof. This is the analogue for cones of the fact that a nonempty subset of $\mathbf{Q}^{n}$ is a convex polytope if and only if it is a bounded polyhedral set [B, Theorem 9.2].

When $S$ spans $\mathbf{Q}^{n}$ we call the halfspaces $H_{i}$ of Theorem 1.1(a) the bounding halfspaces of cone $(S)$. In the terminology of [B] these are the supporting halfspaces which intersect cone $(S)$ in a facet. If $H$ is a bounding halfspace of cone $(S)$ then $H^{0}$ will be called a bounding hyperplane.

1.2. LemmA. Suppose $C=H_{1} \cap H_{2} \cap \cdots \cap H_{r}$ is an intersection of halfspaces in $\mathbf{Q}^{n}$ and that $C$ spans $\mathbf{Q}^{n}$. Then any bounding halfspace of $C$ is equal to some $H_{i}$.

Proof. Suppose $H=\left\{v \in \mathbf{Q}^{n} \mid f(v) \geqslant 0\right\}$ is a bounding halfspace different from each $H_{i}=\left\{v \in \mathbf{Q}^{n} \mid f_{i}(v) \geqslant 0\right\}$. Since $H^{0} \cap C$ contains $n-1$ linearly independent elements, there exists $v \in H^{0} \cap C$ such that $v \notin H_{i}^{0}$. Adding the elements obtained in this way we obtain $u$ with $f(u)=0$ and $f_{i}(u)>0$ for $i=1, \ldots, r$. The idea now is to perturb $u$ slightly to obtain an element $u^{\prime}$ of $C$ which does not lie in $H$. 
There exists $w \in \mathbf{Q}^{n}$ such that $f(w)=-1$. Let $f_{i}(u)=\varepsilon_{i}>0$ and $f_{i}(w)=\delta_{i}$. If $\delta_{i} \geqslant 0$ for $1 \leqslant i \leqslant r$, then $u^{\prime}=u+w$ is the desired element. If $\delta_{i}<0$ for some $i$, let $\lambda=\min \left\{-\varepsilon_{i} / \delta_{i} \mid 1 \leqslant i \leqslant r\right.$ and $\left.\delta_{i}<0\right\}$, so $\lambda>0$.

We set $u^{\prime}=u+\lambda w$. Then it is easily checked that $f_{i}\left(u^{\prime}\right) \geqslant 0$ for $1 \leqslant i \leqslant r$, so $u^{\prime} \in C$, but $f\left(u^{\prime}\right)=-\lambda<0$ so $u^{\prime} \notin H$ and $H$ is not a bounding halfspace of $C$.

1.3. We next characterize certain semigroups which we shall be interested in. An additive abelian semigroup $\Lambda$ is normal if it is finitely generated, cancellative, and whenever $p_{1}, p_{2}, p_{3} \in \Lambda$ with $p_{1}+n p_{2}=n p_{3}$ for a positive integer $n$, there exists $p_{4} \in \Lambda$ with $p_{1}=n p_{4}$. In this situation $\Lambda$ can be embedded in a finitely generated abelian group ( $\mathbf{Z} \Lambda$ for example). However the definition of normality is independent of the embedding. For $\Lambda$ a subsemigroup of $\mathbf{Z}^{n}$, we write $k \Lambda=\operatorname{span}\left\{t^{\lambda} \mid \lambda \in \mathbf{Z}^{n}\right\}$.

Proposition. For a semigroup $\Lambda$ the following are equivalent:

(1) $\Lambda$ is normal.

(2) For any field $k, k \Lambda$ is an integrally closed Noetherian domain.

(3) For some $s \geqslant t \geqslant 0, \Lambda$ is isomorphic to a semigroup of the form $\left(\mathbf{Z}_{+}^{t} \times \mathbf{Z}^{s-t}\right) \cap V$, where $V$ is a subspace of $\mathbf{Q}^{s}$ and under this isomorphism $\operatorname{dim} \mathbf{Q} \Lambda=n$.

(4) For some $t, n \geqslant 0, \Lambda$ is isomorphic to a semigroup of the form $\mathbf{Z}^{n} \cap H_{1}$ $\cap \cdots \cap H_{t}$ where the $H_{i}$ are halfspaces in $\mathbf{Q}^{n}$, and under this isomorphism $\mathbf{Z} \Lambda=\mathbf{Z}^{n}$.

In the proof that $(4) \Rightarrow(3)$ we may take $s=t+n$.

PROof. The proof of the equivalence of (1)-(3) can be adapted from the proof of [Ho, Proposition 1].

(4) $\Rightarrow$ (3). Assume $\Lambda=\mathbf{Z}^{n} \cap H_{1} \cap \cdots \cap H_{t}$ where $H_{i}=\left\{v \in \mathbf{Q}^{n} \mid f_{i}(v) \geqslant 0\right\}$. Define $y_{i}=f_{i}\left(x_{1}, \ldots, x_{n}\right)$ for $i=1, \ldots, t$. Then $y_{i} \in \mathbf{Z}^{+}$for $\left(x_{1}, \ldots, x_{n}\right) \in \Lambda$. The $\operatorname{map}\left(x_{1}, \ldots, x_{n}\right) \rightarrow\left(y_{1}, \ldots, y_{t}, x_{1}, \ldots, x_{n}\right)$ is an isomorphism of $\mathbf{Q} \Lambda$ onto the subspace $V$ of $\mathbf{Q}^{t+n}$ defined by the equations $Y_{i}=f_{i}\left(X_{1}, \ldots, X_{n}\right)$. Clearly the restriction of this map to $\Lambda$ maps $\Lambda$ isomorphically onto $\left(\mathbf{Z}_{+}^{t} \times \mathbf{Z}^{n}\right) \cap V$.

(3) $\Rightarrow$ (4). We assume $\Lambda=\left(\mathbf{Z}_{+}^{t} \times \mathbf{Z}^{s-t}\right) \cap V$ and show $\Lambda$ is isomorphic to a semigroup of the form $\mathbf{Z}^{n} \cap H_{1} \cap \cdots \cap H_{t}$ as in part (4). We can choose a basis $v_{1}, \ldots, v_{n}$ for $\mathbf{Q} \Lambda$ such that $v_{1}, \ldots, v_{n}$ generate $\mathbf{Z} \Lambda$ as a group. Then we define an isomorphism $\phi: \mathbf{Q} \Lambda \rightarrow \mathbf{Q}^{n}$ by $\phi\left(\sum_{i=1}^{n} \lambda_{i} v_{i}\right)=\left(\lambda_{1}, \ldots, \lambda_{n}\right)$. Clearly $\phi$ maps $\mathbf{Z} \Lambda$ isomorphically onto $\mathbf{Z}^{n}$. Let $\psi$ be the composite $\mathbf{Q}^{n} \stackrel{\phi^{-1}}{\rightarrow} \mathbf{Q} \Lambda \subseteq \mathbf{Q}^{s}$. Define $\psi^{*}$ : $\left(\mathbf{Q}^{s}\right)^{*} \rightarrow\left(\mathbf{Q}^{n}\right)^{*}$ by $\left(\psi^{*} g\right)(v)=g(\psi(v))$ for $g \in\left(\mathbf{Q}^{s}\right)^{*}, v \in \mathbf{Q}^{n}$. Let $x_{i} \in\left(\mathbf{Q}^{s}\right)^{*}$ be the coordinate functions defined by $x_{i}(\mu)=\mu_{i}$ for $\mu=\left(\mu_{1}, \ldots, \mu_{s}\right) \in \mathbf{Q}^{s}$ and for $i=1, \ldots, t$ define $f_{i}=\psi^{*} x_{i} \in\left(\mathbf{Q}^{n}\right)^{*}$. Let $H_{i}$ be the halfspace $H_{i}=\left\{u \in \mathbf{Q}^{n} \mid f_{i}(v)\right.$ $\geqslant 0\}$. We claim that $\phi$ maps $\Lambda$ isomorphically onto $\mathbf{Z}^{n} \cap H_{1} \cap \cdots \cap H_{t}$.

Certainly $\phi(\Lambda) \subseteq \mathbf{Z}^{n}$, since $\phi(\mathbf{Z} \Lambda)=\mathbf{Z}^{n}$. If $\lambda \in \Lambda$, then $f_{i}(\phi(\lambda))=$ $\left(\psi^{*} x_{i}\right)(\phi(\lambda))=x_{i}(\psi(\phi(\lambda)))=x_{i}(\lambda) \geqslant 0$ since $\psi \circ \phi$ is just the inclusion $\mathbf{Q} \Lambda \subseteq \mathbf{Q}^{s}$. Hence $\phi(\lambda) \in H_{i}$ and $\phi(\Lambda) \subseteq \mathbf{Z}^{n} \cap H_{1} \cap \cdots \cap H_{t}$.

Finally, given $\mu \in \mathbf{Z}^{n} \cap H_{1} \cap \cdots \cap H_{t}$ there exists $\lambda \in \mathbf{Z} \Lambda$ with $\phi(\lambda)=\mu$, since $\phi: \mathbf{Z} \Lambda \rightarrow \mathbf{Z}^{n}$ is an isomorphism. The computation above shows that $x_{i}(\lambda) \geqslant 0$ for $i=1, \ldots, t$ so $\lambda \in \Lambda$ and $\phi: \Lambda \rightarrow \mathbf{Z}^{n} \cap H_{1} \cap \cdots \cap H_{t}$ is the desired isomorphism.

The maps $\phi, \psi$, and $\psi^{*}$ constructed above will play an important role in $\S 2$. 
1.4. We now interpret our work in terms of rings of invariants of tori. Assume that $G=\mathrm{GL}(1, k)^{r}$ is a torus acting on $\mathcal{O}\left(U_{\mathbf{s}}\right)=k\left[T_{1}^{ \pm 1}, \ldots, T_{s}^{ \pm 1}\right]$ so that for $g=$ $\left(g_{1}, \ldots, g_{r}\right) \in G$

$$
g \cdot T_{j}=\prod_{i=1}^{r} g_{i}^{a_{i}} T_{j} \quad \text { where } a_{i j} \in \mathbf{Z} .
$$

It is easily checked that a monomial $T^{\lambda}$ is fixed by $G$ if and only if $m_{i}(\lambda)=0$ for $1 \leqslant i \leqslant r$ where

$$
m_{i}=\sum_{j=1}^{s} a_{i j} x_{j} \in \cdot\left(\mathbf{Q}^{s}\right)^{*} .
$$

It follows that $\mathcal{O}\left(U_{\beta}\right)^{G}=k \Lambda_{\beta}$, a semigroup algebra, where

$$
\Lambda_{\beta}=\left\{\lambda \in \mathbf{Z}^{s} \mid m_{i}(\lambda)=0 \text { for } 1 \leqslant i \leqslant r, \lambda_{j} \geqslant 0 \text { if } j \notin \beta\right\} .
$$

Let $V=\left\{\lambda \in \mathbf{Q}^{s} \mid m_{i}(\lambda)=0\right.$ for $\left.1 \leqslant i \leqslant r\right\}$, and $H_{i}=\left\{\lambda \in \mathbf{Q}^{s} \mid \lambda_{i} \geqslant 0\right\}$. Then $\Lambda_{\beta}=\mathbf{Z}^{s} \cap V \cap \bigcap_{j \notin \beta} H_{j}$ is a semigroup of the type described in statement (3) of Proposition 1.3, and $\mathcal{O}\left(U_{\mathrm{s}}\right)^{G}=k \Lambda_{\mathrm{s}}$ where $\Lambda_{\mathrm{s}}=\mathbf{Z}^{s} \cap V$.

Conversely given $\Lambda_{\beta}, \Lambda_{\mathrm{s}}$ of this form, we may assume the vector space $V$ is the solution space of a system of linear equations as in (2), define an action of a torus $G$ on $\mathcal{O}\left(U_{\mathrm{s}}\right)$ as in (1), and then we obtain $\mathcal{O}\left(U_{\mathrm{s}}\right)^{G}=k \Lambda_{\mathrm{s}}$ and $\mathcal{O}\left(U_{\beta}\right)^{G}=k \Lambda_{\beta}$.

It is often useful to regard our torus $G$ as a subtorus of the torus $\Gamma=\operatorname{GL}(1)^{s}$ which consists of all invertible linear transformations represented by diagonal matrices on the basis $T_{1}, \ldots, T_{s}$. For $g=\left(g_{1}, \ldots, g_{s}\right) \in \Gamma$ we have $g T_{i}=g_{i} T_{i}$, so if we define $T_{i}(g)=g_{i}$ then $T_{1}, \ldots, T_{s}$ generate the character group $M=M(\Gamma)$ of $\Gamma$ and

$$
g \cdot T^{\lambda}=T^{\lambda}(g) T^{\lambda} \quad \text { for } g \in \Gamma, \lambda \in \mathbf{Z}^{s} .
$$

The correspondence $\lambda \leftrightarrow T^{\lambda}$ allows us to switch between additive and multiplicative notation for $M$. We obtain an induced action of $\Gamma$ on $\mathbf{Z}^{s}$ by

$$
g \cdot \lambda=T^{\lambda}(g) \lambda \quad \text { for } g \in \Gamma, \lambda \in \mathbf{Z}^{s} .
$$

If $N=N(\Gamma)$ is the group of one parameter subgroups of $\Gamma$ there is a pairing (, ): $N \times M \rightarrow \mathbf{Z}$ defined by

$$
T^{\lambda}(x(g))=g^{(x, \lambda)} \quad \text { for } g \in k, x \in N, \lambda \in M .
$$

Let $x_{1}, \ldots, x_{s}$ be the basis for $N$ such that $T_{i}\left(x_{j}(g)\right)=g^{\delta_{i j}}$, for all $g \in k$, where $\delta_{i j}$ is the Kronecker delta. We also write $x(\lambda)=(x, \lambda)$. Then $x_{i}(\lambda)=\lambda_{i}$ and $x_{1}, \ldots, x_{s}$ can be viewed as coordinate functions on $\mathbf{Z}^{s}$.

Lemma. There exists a subset $\beta^{\prime}$ of $\mathbf{s}$ containing $\beta$ such that $\mathcal{O}\left(U_{\beta}\right)^{G}=\mathcal{O}\left(U_{\beta^{\prime}}\right)^{G}$ and the distinct bounding halfspaces of $\mathbf{Q}^{+} \Lambda_{\beta}=\mathbf{Q}^{+} \Lambda_{\beta^{\prime}}$ are precisely the halfspaces $\mathbf{Q} \Lambda_{\beta} \cap H_{j}$ with $j \notin \beta^{\prime}$.

Proof. By Lemma 1.2 any bounding halfspace of $\mathbf{Q}^{+} \Lambda_{\beta}$ has the form $\mathbf{Q} \Lambda_{\beta} \cap H_{j}$ where $j \notin \beta$. Simply choose a subset $\beta^{\prime}$ containing $\beta$ so the distinct bounding halfspaces have the form $\mathbf{Q} \Lambda_{\beta} \cap H_{j}$ where $j \notin \beta^{\prime}$. 
1.5. In the situations described above where $\mathcal{O}\left(U_{\mathrm{s}}\right)^{G}=k \Lambda_{\mathrm{s}}, \mathcal{O}\left(U_{\beta}\right)^{G}=k \Lambda_{\beta}$, it is possible to have $\mathbf{Z} \Lambda_{\beta}$ strictly contained in the group $\Lambda_{\mathrm{s}}=\mathbf{Z}^{s} \cap V$. However if we are given $\Lambda_{\beta}=\mathbf{Z}^{s} \cap V \cap \cap_{j \notin \beta} H_{j}$ for some subspace $V$ of $\mathbf{Q} \Lambda_{\beta}$ then since $\Lambda_{\beta} \subseteq$ $\mathbf{Q} \Lambda_{\beta} \subseteq V$ we obtain $\Lambda_{\beta} \subseteq \mathbf{Z}^{s} \cap \mathbf{Q} \Lambda_{\beta} \cap \cap_{j \notin \beta} H_{j} \subseteq \mathbf{Z}^{s} \cap V \cap \cap_{j \notin \beta} H_{j}=\Lambda_{\beta}$. Hence $\Lambda_{\beta}=\mathbf{Z}^{s} \cap \mathbf{Q} \Lambda_{\beta} \cap \cap_{j \notin \beta} H_{j}$, and we could replace $V$ by $\mathbf{Q} \Lambda_{\beta}$ in the definition of $\Lambda_{\beta}$. We also note

Lemma. $\mathbf{Z} \Lambda_{\beta}=\mathbf{Z}^{s} \cap \mathbf{Q} \Lambda_{\beta}$.

Proof. If $\lambda \in \mathbf{Z}^{s} \cap \mathbf{Q} \Lambda_{\beta}$, there is an integer $p \geqslant 1$ such that $p \lambda \in \mathbf{Z} \Lambda_{\beta}$. Hence $p \lambda=\lambda_{1}-\lambda_{2}$ for $\lambda_{1}, \lambda_{2} \in \Lambda_{\beta}$, so $p \lambda=p \lambda_{1}-\lambda_{3}$ where $\lambda_{3}=\lambda_{2}+(p-1) \lambda_{1} \in$ $\Lambda_{\beta} \cap p \mathbf{Z}^{s}=p \Lambda_{\beta}$. Therefore $\lambda_{3}=p \lambda_{4}$ for some $\lambda_{4} \in \Lambda_{\beta}$ and $\lambda=\lambda_{1}-\lambda_{4} \in \mathbf{Z} \Lambda_{\beta}$. Since the inclusion $\mathbf{Z} \Lambda_{\beta} \subseteq \mathbf{Z}^{s} \cap \mathbf{Q} \Lambda_{\beta}$ is obvious this proves the result.

1.6. Applying the procedure outlined after Proposition 1.3 to $\Lambda_{\beta}$ and $\mathbf{Z} \Lambda_{\beta}$ we obtain

COROLLARY. Given an action of a torus $G$ on $\mathcal{O}\left(U_{\mathrm{s}}\right)$ we may extend the action of $G$ to a larger torus $G^{\prime}$ so that $\mathcal{O}\left(U_{\beta}\right)^{G^{\prime}}=k \Lambda_{\beta}$ and $\mathcal{O}\left(U_{\mathrm{s}}\right)^{G^{\prime}}=k \mathbf{Z} \Lambda_{\beta}$.

There is no loss of generality in assuming that the torus $G$ acts faithfully on $\mathcal{O}\left(U_{\mathrm{s}}\right)$ and we shall always do so. This is equivalent to requiring that the equations $m_{j}(x)$, $j=1, \ldots, r$, are linearly independent. If $\mathcal{O}\left(U_{\beta}\right)^{G}=k \Lambda_{\beta}$, then the Krull dimension of $\mathcal{O}\left(U_{\beta}\right)^{G}$ is equal to the rank of $\mathbf{Z} \Lambda_{\beta}$. Thus by linear algebra

$$
\operatorname{dim} \mathcal{O}\left(U_{\beta}\right)-\operatorname{dim} G=s-r=\operatorname{dim} \mathcal{O}\left(U_{\mathrm{s}}\right)^{G} \geqslant \operatorname{dim} \mathcal{O}\left(U_{\beta}\right)^{G} .
$$

Here $\operatorname{dim} G=r$ is the dimension of $G$ as an algebraic group, and the dimensions of the rings are their Krull dimensions. We say that $G$ satisfies the dimension equality on $\mathcal{O}\left(U_{\beta}\right)$ provided $\operatorname{dim} \mathcal{O}\left(U_{\beta}\right)^{G}=\operatorname{dim} \mathcal{O}\left(U_{\beta}\right)-\operatorname{dim} G$. By Lemma 1.5 this is equivalent to the requirement that $\mathbf{Z} \Lambda_{\beta}=\Lambda_{\mathrm{s}}$. By Corollary 1.6 we may assume the dimension equality holds by embedding $G$ in a larger torus without changing $\mathcal{O}\left(U_{\beta}\right)^{G}$.

1.7. Lemma. Suppose $\Lambda=\left(\mathbf{Z}_{+}^{r} \times \mathbf{Z}^{s-r}\right) \cap V$ and that $\mathbf{Q} \Lambda \cap \operatorname{ker} x_{1}$ is a bounding hyperplane of $\mathbf{Q}^{+} \Lambda$. If $x_{1}(\mathbf{Z} \Lambda)=a \mathbf{Z}$ for some $a>0$ then there exists $\lambda \in \Lambda$ such that $x_{1}(\lambda)=a$.

Proof. By assumption $x_{1}\left(\lambda^{\prime}\right)=a$ for some $\lambda^{\prime} \in \mathbf{Z} \Lambda$. Let $H_{j}=\left\{\lambda \in \mathbf{Q}^{s} \mid x_{j}(\lambda)\right.$ $\geqslant 0\}$. For $2 \leqslant j \leqslant r$ we consider three cases which may arise. If $\mathbf{Q} \Lambda \subseteq H_{j}^{0}$ then $x_{j}\left(\lambda^{\prime}\right)=0$; in this case let $u_{j}=0$. In the other cases $\mathbf{Q} \Lambda \cap H_{j}^{0}$ has codimension one in $\mathbf{Q} \Lambda$.

Suppose next that $H_{1}^{0} \cap \mathbf{Q} \Lambda=H_{j}^{0} \cap \mathbf{Q} \Lambda$. Then $H_{1} \cap \mathbf{Q} \Lambda=H_{j} \cap \mathbf{Q} \Lambda$ or $H_{1} \cap$ $\mathbf{Q} \Lambda=\left(-H_{j}\right) \cap \mathbf{Q} \Lambda$. Since $\mathbf{Q} \Lambda \nsubseteq H_{1}^{0}$ there exists $\mu \in \Lambda$ with $x_{1}(\mu)>0$, and so $\mu \in H_{1} \cap \mathbf{Q} \Lambda$ but $\mu \notin\left(-H_{j}\right) \cap \mathbf{Q} \Lambda$. Therefore $H_{1} \cap \mathbf{Q} \Lambda=H_{j} \cap \mathbf{Q} \Lambda$, so $x_{j}\left(\lambda^{\prime}\right)$ $>0$ in this case and we set $u_{j}=0$.

Finally suppose that $H_{1}^{0} \cap \mathbf{Q} \Lambda$ and $H_{j}^{0} \cap \mathbf{Q} \Lambda$ are distinct subspaces of codimension one in $\mathbf{Q} \Lambda$. Then $H_{1}^{0} \cap \mathbf{Q} \Lambda \nsubseteq H_{j}^{0}$ and since $H_{1}^{0} \cap \Lambda$ spans $H_{1}^{0} \cap \mathbf{Q} \Lambda$, there exists $u_{j} \in H_{1}^{0} \cap \Lambda$ with $u_{j} \notin H_{j}^{0}$. Therefore $x_{1}\left(u_{j}\right)=0$ and $x_{j}\left(u_{j}\right)>0$. 
Set $u=u_{2}+\cdots+u_{r} \in \Lambda$. Then $x_{1}(u)=0$ and in the first two cases $x_{j}\left(\lambda^{\prime}\right) \geqslant 0$ and $x_{j}(u) \geqslant 0$, while in the last case $x_{j}(u)>0$. If we set $\lambda=\lambda^{\prime}+N u$ for a sufficiently large positive integer $N$, then $\lambda$ will satisfy the conditions of the lemma.

1.8. We make one further reduction. Suppose $\Lambda_{\beta}=\mathbf{Z}^{s} \cap V \cap \cap_{j \notin \beta} H_{j}$ and the groups $\mathbf{Z} \Lambda_{\beta}$ are equal for all $\beta \in \Delta$. For all $j$ such that $\mathbf{Q} \Lambda \nsubseteq \operatorname{ker} x_{j}$, let $x_{j}\left(\mathbf{Z} \Lambda_{\beta}\right)$ $=a_{j} \mathbf{Z}$ where $a_{j} \geqslant 1$. We define an isomorphism $\phi: \mathbf{Q}^{s} \rightarrow \mathbf{Q}^{s}$ by $\phi\left(\lambda_{1}, \ldots, \lambda_{s}\right)=$ $\left(\mu_{1}, \ldots, \mu_{s}\right)$ where $\mu_{j}=\lambda_{j}$ if $\mathbf{Q} \Lambda \subset \operatorname{ker} x_{j}$ and $\mu_{j}=\lambda_{j} / a_{j}$ otherwise. Let $\psi=\phi^{-1}$ and as in the proof of Proposition 1.3 define $\psi^{*}:\left(\mathbf{Q}^{s}\right)^{*} \rightarrow\left(\mathbf{Q}^{s}\right)^{*}$ by $\left(\psi^{*} f\right)(v)=$ $f(\psi(v))$. Let $V^{\prime} \subseteq \mathbf{Q}^{s}$ be the solution space to the equations $m_{j}^{\prime}=\psi^{*} m_{j}, 1 \leqslant j \leqslant r$. Then $\phi$ maps $\Lambda_{\beta}$ isomorphically onto the semigroup $\Lambda_{\beta}^{\prime}=\mathbf{Z}^{s} \cap V^{\prime} \cap \bigcap_{j \notin \beta} H_{j}$ and $x_{j}\left(\mathbf{Z} \Lambda_{\beta}\right)=\mathbf{Z}$ for all $j \notin \beta$. In terms of fixed rings of tori we have shown the following lemma.

Lemma. There exists an action of $G^{\prime} \cong G$ on $k^{s}$ such that for all $\beta \in \Delta, \mathcal{O}\left(U_{\beta}\right)^{G^{\prime}}=$ $k \Lambda_{\beta}^{\prime} \cong k \Lambda_{\beta}$ and for all $j \notin \beta, x_{j}\left(\mathbf{Z} \Lambda_{\beta}^{\prime}\right)=\mathbf{Z}$.

1.9. If $G$ satisfies the dimension equality, the reduction achieved in the previous lemma has an interesting interpretation in terms of pseudoreflections.

Lemma. Assume $G$ satisfies the dimension equality on $\mathcal{O}\left(U_{\beta}\right)$. If $\mathcal{O}\left(U_{\beta}\right)^{G}=k \Lambda_{\beta}$ then $x_{j}\left(\mathbf{Z} \Lambda_{\beta}\right)=\mathbf{Z}$ if and only if $G$ contains no nonidentity pseudoreflection fixing the hyperplane defined by $x_{j}=0$.

Proof. Without loss of generality $j=1$. If $G$ contains a pseudoreflection of the form $(\omega, 1, \ldots, 1)$ where $\omega$ is a primitive $m$ th root of unity, $m>1$, then $k \Lambda_{\beta}=$ $\mathcal{O}\left(U_{\beta}\right)^{G} \subseteq \mathcal{O}\left(U_{\mathbf{s}}\right)^{G} \subseteq k\left[T_{1}^{ \pm m}, T_{2}^{ \pm 1}, \ldots, T_{s}^{ \pm 1}\right]$ and $x_{1}\left(\mathbf{Z} \Lambda_{\beta}\right) \subseteq m \mathbf{Z}$.

Conversely, assume $x_{1}\left(\mathbf{Z} \Lambda_{\beta}\right)=m \mathbf{Z}$ where $m>1$. For $g \in \Gamma, \lambda \in \mathbf{Z}^{s}$ we have $g \cdot T^{\lambda}=T^{\lambda}(g) T^{\lambda}$. Hence $\mathcal{O}\left(U_{\mathrm{s}}\right)^{G}=\operatorname{Span}\left\{T^{\lambda} \mid \lambda \in \mathbf{Z}^{s}, G \subseteq \operatorname{Ker} T^{\lambda}\right\}$. Since $x_{1}\left(\mathbf{Z} \Lambda_{\beta}\right)=m \mathbf{Z}$ and $\mathbf{Z} \Lambda_{\beta}=\Lambda_{\mathrm{s}}$ by the dimension equality, we have $\mathcal{O}\left(U_{\mathrm{s}}\right)^{G} \subseteq$ $k\left[T_{1}^{ \pm m}, T_{2}^{ \pm 1}, \ldots, T_{s}^{ \pm 1}\right]$. Hence the pseudoreflection $g=(\omega, 1, \ldots, 1)$ fixes $\mathcal{O}\left(U_{\mathrm{s}}\right)^{G}$ where $\omega$ is a primitive $m$ th root of unity. Therefore $g \in \operatorname{Ker} T^{\lambda}$ for all $\lambda \in \mathbf{Z}^{s}$ such that $G \subseteq \operatorname{Ker} T^{\lambda}$. However by [Hu, Proposition 16.1] $G=\cap \operatorname{Ker} T^{\lambda}$ where the intersection is over those kernels of characters which contain $G$. Hence $g \in G$ as required.

1.10. EXAmple. If $G$ does not satisfy the dimension equality on $\mathcal{O}\left(U_{\beta}\right)$ the above result may fail to hold.

Let $G=\mathrm{GL}(1, k)^{2}$ and let $G$ act on $K=k\left[T_{1}, T_{2}, T_{3}, T_{4}\right]$ by $g \cdot T_{j}=\Pi_{i} g_{i}^{a_{i}} T_{j}$ where

$$
\left(a_{i j}\right)=\left(\begin{array}{cccc}
1 & -1 & 0 & -2 \\
-1 & 0 & -1 & 2
\end{array}\right)
$$

Then $T^{\lambda} \in K^{G}$ if and only if $\lambda$ satisfies

$$
\begin{aligned}
\lambda_{1}-\lambda_{2}-2 \lambda_{4} & =0 \quad \text { and } \quad \lambda_{i} \geqslant 0, \\
-\lambda_{1}-\lambda_{3}+2 \lambda_{4} & =0 .
\end{aligned}
$$

Adding these equations we find $\lambda_{2}+\lambda_{3}=0$. Therefore $\lambda_{2}=\lambda_{3}=0$, and $K^{G}=k \Lambda$ where $\Lambda=\mathbf{Z}_{+}(2,0,0,1)$. Hence $x_{1}(\mathbf{Z} \Lambda)=2 \mathbf{Z}$. However if $g \in G$ is a pseudoreflection fixing the hyperplane defined by $x_{1}=0$, then from $g \cdot T_{2}=T_{2}$ and $g \cdot T_{3}=T_{3}$, it follows that $g_{1}=g_{2}=1$ so $g=1$. 


\section{Rings of differential operators.}

2.0. We explain the notation for halfspaces and hyperplanes which we will be using from now on. Let $x_{i}$ be the coordinate functions on $\mathbf{Q}^{s}, \mathscr{H}_{i}=\left\{\lambda \in \mathbf{Q}^{s} \mid x_{i}(\lambda)\right.$ $\geqslant 0\}$, and $\mathscr{H}_{i}^{0}=\operatorname{ker} x_{i}$. Let $f_{i}$ be the restriction of $x_{i}$ to $\mathbf{Q} \Lambda, H_{i}=\mathscr{H}_{i} \cap \mathbf{Q} \Lambda$, and $H_{i}^{0}=\mathscr{H}_{i}^{0} \cap \mathbf{Q} \Lambda=\operatorname{ker} f_{i}$. Identifying $\mathbf{Q} \Lambda$ with $\mathbf{Q}^{n}$ by means of the isomorphism $\phi$, we have $f_{i}=\psi^{*} x_{i}$ as in Proposition 1.3.

We are now able to formulate Theorem B. Suppose the torus $G=\operatorname{GL}(1)^{r}$ acts on $Y=\cup_{\beta \in \Delta} U_{\beta}$ as in the introduction. For each $\beta \in \Delta, \mathcal{O}\left(U_{\beta}\right)^{G}=k \Lambda_{\beta}$ where

$$
\Lambda_{\beta}=\left\{\lambda \in \mathbf{Z}^{s} \mid m_{i}(\lambda)=0 \text { for } 1 \leqslant i \leqslant r, \lambda_{j} \geqslant 0 \text { if } j \notin \beta\right\}
$$

as described in $\S 1$. Since for each $\beta \in \Delta, k \Lambda_{\beta}$ has the same quotient field, the groups $\mathbf{Z} \Lambda_{\beta}$ are equal for $\beta \in \Delta$, and we write $\mathbf{Z} \Lambda=\mathbf{Z} \Lambda_{\beta}$ and similarly $\mathbf{Q} \Lambda=\mathbf{Q} \Lambda_{\beta}$ for any $\beta \in \Delta$.

The conditions for $\theta$ to be surjective are expressed in terms of the relationship between the halfspaces in $\mathbf{Q}^{s}$ determined by the coordinate hyperplanes and their restriction to the subspace $\mathbf{Q} \Lambda$. By Lemma 1.2 if $\beta \in \Delta$, any bounding halfspace of $\mathbf{Q}^{+} \Lambda_{\beta}$ has the form $\mathbf{Q} \Lambda \cap \mathscr{H}_{j}$ where $\mathbf{Q} \Lambda \nsubseteq \mathscr{H}_{j}^{0}$ and $j \notin \beta$. We call the set of such halfspaces as $\beta$ runs over $\Delta$, the induced halfspaces of $\mathbf{Q} \Lambda$. That is set $\alpha=\left\{j \in \mathbf{s} \mid \mathbf{Q} \Lambda \nsubseteq \mathscr{H}_{j}^{0}\right.$, and $\left.j \notin \bigcap_{\beta \in \Delta} \beta\right\}$. Then an induced halfspace of $\mathbf{Q} \Lambda$ is of the form $\mathbf{Q} \Lambda \cap \mathscr{H}_{j}$ with $j \in \alpha$. We say that a differential operator $d \in D\left(U_{\mathrm{s}}\right)$ is homogeneous if for every monomial $f \in \mathcal{O}\left(U_{\mathrm{s}}\right)$ we have $d \cdot f=\lambda f$ for some $\lambda \in k$, depending on $f$.

THForem B. (1) The map $\theta: D(Y)^{G} \rightarrow D\left(Y_{\Delta} / G\right)$ is surjective if and only if

(a) The induced halfspaces of $\mathbf{Q} \Lambda$ are distinct.

(b) Every induced halfspace of $\mathbf{Q} \Lambda$ is a bounding halfspace of $\mathbf{Q}^{+} \Lambda_{\beta}$ for some $\beta \in \Delta$.

(c) If $\mathbf{Q} \Lambda \cap \mathscr{H}_{i}$ is an induced halfspace of $\mathbf{Q} \Lambda$ then $x_{i}(\mathbf{Z} \Lambda)=\mathbf{Z}$.

(2) Let $J$ be the left ideal of $D\left(U_{\mathrm{s}}\right)^{G}$ generated by all first order homogeneous differential operators which vanish on $k \mathbf{Z} \Lambda$. Then $\operatorname{Ker} \theta=J \cap D(Y)^{G}$.

We give some examples to illustrate conditions (a)-(c) of Theorem B part (1) at the end of this section. We also show that the definition of $D\left(Y_{\Delta} / G\right)$ may depend on $\Delta$ and not just on $Y$ and $G$.

In Proposition 3.7, we show how conditions (a)-(c) of (1) can be achieved without changing $D\left(Y_{\Delta} / G\right)$ up to isomorphism.

To prove Theorem $\mathrm{B}$ we shall obtain explicit descriptions of the rings $D(Y)^{G}$, $D\left(Y_{\Delta} / G\right)$, and the map $\theta$.

2.1. LEMMA. If $K$ is a commutative $k$-algebra and $\mathscr{C}$ a multiplicatively closed subset of $K$ consisting of regular elements, then any differential operator on $K$ has a unique extension to $K_{\mathscr{C}}$. In addition $D(K)=\left\{x \in D\left(K_{\mathscr{C}}\right) \mid x \cdot K \subseteq K\right\}$.

Proof. See [H, Lemma 2].

2.2. Let $k\left[Q_{1}, \ldots, Q_{s}, P_{1}, \ldots, P_{s}\right]$ be the $s$ th Weyl algebra over $k$ with generators $Q_{j}, P_{i}$ satisfying $\left[P_{i}, Q_{j}\right]=\delta_{i j}$ where $[a, b]=a b-b a$. We regard this ring as the ring of differential operators on $k\left[T_{1}, \ldots, T_{s}\right]$ where $Q_{i}$ acts as multiplication by $T_{i}$ 
and $P_{i}$ as $\partial / \partial T_{i}$. Since $\mathcal{O}(Y)=\bigcap_{\beta \in \Delta} \mathcal{O}\left(U_{\beta}\right)=k\left[T_{1}, \ldots, T_{s}, T_{i}^{-1} ; i \in \bigcap_{\beta \in \Delta} \beta\right]$ we can regard $D(Y)$ as a subalgebra of $D\left(U_{\mathrm{s}}\right)=k\left[Q_{1}^{ \pm 1}, \ldots, Q_{s}^{ \pm 1}, P_{1}, \ldots, P_{s}\right]$ by Lemma 2.1. It is easy to show that $D\left(U_{\mathrm{s}}\right)=\oplus_{\lambda \in \mathbf{Z}^{\mathrm{s}}} D\left(U_{\mathrm{s}}\right)(\lambda)$ where $D\left(U_{\mathrm{s}}\right)(\lambda)=\{x \in$ $D\left(U_{\mathrm{s}}\right) \mid\left[Q_{i} P_{i}, x\right]=\lambda_{i} x$ for $\left.1 \leqslant i \leqslant s\right\}$. We denote by $A$ the subring $A=D\left(U_{\mathrm{s}}\right)(0)=$ $k\left[Q_{1} P_{1}, \ldots, Q_{s} P_{s}\right]$ of $D\left(U_{\mathrm{s}}\right)$. Then $A$ is exactly the set of homogeneous differential operators on $\mathcal{O}\left(U_{\mathbf{s}}\right)$.

Lemma. Let $R$ be a subring of $D\left(U_{\mathrm{s}}\right)$ containing $A$. If $I$ is an ideal of $R$ then $I=\oplus_{\lambda \in \mathbf{Z}^{\mathrm{s}}} I(\lambda)$ where $I(\lambda)=\left\{x \in I \mid\left[Q_{i} P_{i}, x\right]=\lambda_{i} x\right.$ for $\left.1 \leqslant i \leqslant s\right\}$.

Proof. Let $\Gamma=\mathrm{GL}(1)^{s}$. Then $\Gamma$ acts rationally on $\mathcal{O}(\Gamma)=\mathcal{O}\left(U_{\mathrm{s}}\right)$ and $D(\Gamma)=$ $D\left(U_{\mathrm{s}}\right)$. Furthermore the Lie algebra Lie $\Gamma$ of $\Gamma$ can be identified, as differential operators on $\mathcal{O}(\Gamma)$, with $k Q_{1} P_{1}+\cdots+k Q_{s} P_{s}$. The action of $\Gamma$ on $D\left(U_{\mathrm{s}}\right)$ gives the following action of Lie $\Gamma$ :

$$
\xi \cdot d=[\xi, d] \text { for } \xi \in \operatorname{Lie} \Gamma, d \in D\left(U_{\mathrm{s}}\right) .
$$

Therefore, if $R$ is a subring of $D\left(U_{\mathrm{s}}\right)$ containing $A$, and $I$ an ideal of $R$, then $I$ and $R$ are stable under Lie $\Gamma$ and so are $\Gamma$-submodules of $D\left(U_{\mathrm{s}}\right)$. Identifying the character group of $\Gamma$ with $\mathbf{Z}^{s}$, we have $I=\oplus_{\lambda \in \mathbf{Z}^{s}} I(\lambda)$ with $I(\lambda)$ as above, by standard results.

We note also that $R(\lambda) \subseteq Q^{\lambda} A$ and $R(0)=A$.

2.3. We next obtain a description of $D(k \Lambda)$ where $\Lambda$ is an arbitrary subsemigroup of $\mathbf{Z}^{s}$. There is no loss of generality in assuming $\mathbf{Z} \Lambda=\mathbf{Z}^{s}$. In $k \Lambda$ set $\mathscr{C}=\left\{T^{\lambda} \mid \lambda \in\right.$ $\Lambda$ \}. Then $k \Lambda_{\mathscr{C}}=\mathcal{O}\left(U_{\mathrm{s}}\right)=k\left[T_{1}{ }^{ \pm 1}, \ldots, T_{s}^{ \pm 1}\right]$. We view $D(k \Lambda)$ as a subring of $D\left(U_{\mathrm{s}}\right)$. Note that for $\mu \in \mathbf{Z}^{s}$ we have $Q_{i} P_{i} \cdot T^{\mu}=\mu_{i} T^{\mu}$.

Let $A_{\mathbf{Q}}=\mathbf{Q}\left[Q_{1} P_{1}, \ldots, Q_{s} P_{s}\right]$ so $A=A_{\mathbf{Q}} \otimes_{\mathbf{Q}} k$. Elements of $A$ define polynomial functions from $\mathbf{Z}^{s} \subseteq \mathbf{Q}^{s}$ to $k$ by the rule

$$
Q_{i} P_{i}(\mu)=\mu_{i} \quad \text { for } \mu \in \mathbf{Z}^{s} .
$$

With this definition we have for $f \in A$

$$
f\left(Q_{1} P_{1}, \ldots, Q_{s} P_{s}\right) \cdot T^{\mu}=f(\mu) \cdot T^{\mu} \quad \text { for } \mu \in \mathbf{Z}^{s} .
$$

Since we have identified $A_{\mathbf{Q}}$ with the symmetric algebra on $\left(\mathbf{Q}^{s}\right)^{*}$, we can now regard an element $f=f\left(x_{1}, \ldots, x_{s}\right) \in\left(\mathbf{Q}^{s}\right)^{*}$ as an element $f=f\left(Q_{1} P_{1}, \ldots, Q_{s} P_{s}\right)$ of $A_{\mathbf{Q}}$.

For a subset $\Omega$ of $\mathbf{Q}^{s}$ we write $\operatorname{ann}_{A} \mathbf{Q}=\{f \in A \mid f(w)=0$ for all $w \in \Omega\}$. Clearly ann $A=\operatorname{ann}_{A} \bar{\Omega}$ where $\bar{\Omega}$ denotes the Zariski closure of $\Omega$.

THEOREM. Let $\Lambda$ be a subsemigroup of $\mathbf{Z}^{s}$ with $\mathbf{Z} \Lambda=\mathbf{Z}^{s}$. Then

$$
D(k \Lambda)=\bigoplus_{\lambda \in \mathbf{Z}^{s}} D(k \Lambda)(\lambda)
$$

where $D(k \Lambda)(\lambda)=Q^{\lambda} \operatorname{ann}_{A} \Omega(\lambda)$ and $\Omega(\lambda)=\{\mu \in \Lambda \mid \mu+\lambda \notin \Lambda\}$.

Proof. The direct sum decomposition is immediate from Lemma 2.2. It is also clear that $D(k \Lambda)(\lambda) \subset Q^{\lambda} A$. If $f \in A$, then $Q^{\lambda} f \cdot T^{\mu}=f(\mu) T^{\mu+\lambda}$. By Lemma 2.1, $Q^{\lambda} f \in A$ if and only if $f$ vanishes on $\Omega(\lambda)$. This proves the result. 
2.4. We can regard $\mathcal{O}(Y)=k\left[T_{1}, \ldots, T_{s}, T_{i}^{-1}: i \in \bigcap_{\beta \in \Delta} \beta\right]$ as a semigroup algebra $k \Sigma$ where $\Sigma$ is the semigroup $\Sigma=\left\{\sigma \in \mathbf{Z}^{s} \mid \sigma_{i} \geqslant 0\right.$ if $\left.i \notin \bigcap_{\beta \in \Delta} \beta\right\}$. Let $\Omega(\lambda)=$ $\{\mu \in \Sigma \mid \lambda+\mu \notin \Sigma\}$. Then we have $D(Y)=\oplus_{\lambda \in \mathbf{Z}^{\prime}} D(Y)(\lambda)$ where $D(Y)(\lambda)=$ $Q^{\lambda} \operatorname{ann}_{A} \Omega(\lambda)$.

Now $D\left(U_{\mathrm{s}}\right)$ is the twisted group algebra of the group $M=\left\langle Q_{1}^{ \pm 1}, \ldots, Q_{s}^{ \pm 1}\right\rangle$ over the subring $D\left(U_{\mathrm{s}}\right)(0)=A$. As explained in the introduction the action of $G$ on $\mathcal{O}\left(U_{\mathrm{s}}\right)$ extends to an action of $G$ on $D\left(U_{\mathrm{s}}\right)$ by the rule

$$
(g \cdot d)(f)=g\left(d\left(g^{-1} f\right)\right)
$$

for $f \in \mathcal{O}\left(U_{\mathrm{s}}\right), d \in D\left(U_{\mathrm{s}}\right)$, and $g \in G$. A simple computation shows that for $g=\left(g_{1}, \ldots, g_{r}\right) \in G$ we have

$$
g \cdot Q_{j}=\prod_{i=1}^{r} g_{i}^{a_{\prime \prime}} Q_{j}
$$

and

$$
g \cdot P_{j}=\prod_{i=1}^{r} g_{i}^{-a_{i}, P_{j}} .
$$

It follows from this that the subring $A$ is invariant under $G$ and that $D\left(U_{\mathrm{s}}\right)^{G}$ is the twisted group algebra of the subgroup $M_{1}$ of $G$-fixed elements of $M$ over $A$. It is easy to see that $Q^{\lambda} \in M$ is $G$-fixed if and only if $\lambda \in \mathbf{Z}^{s} \cap V$, and thus we obtain

$$
D\left(U_{\mathrm{s}}\right)^{G}=\bigoplus_{\lambda \in \mathbf{Z}^{\prime} \cap V} D\left(U_{\mathrm{s}}\right)(\lambda) .
$$

Since $D(Y)^{G}=D(Y) \cap D\left(U_{\mathrm{s}}\right)^{G}$ we obtain the following result.

THEOREM.

$$
D(Y)^{G}=\bigoplus_{\lambda \in \mathbf{Z}^{\prime} \cap V} D(Y)^{G}(\lambda)
$$

where $D(Y)^{G}(\lambda)=Q^{\lambda} \operatorname{ann}_{A} \overline{\Omega(\lambda)}$, and $\Omega(\lambda)=\{\mu \in \Sigma \mid \mu+\lambda \notin \Sigma\}$.

2.5. By identifying $\mathbf{Z} \Lambda$ with $\mathbf{Z}^{n}$ we have $k \mathbf{Z} \Lambda=k\left[t_{1}^{ \pm 1}, \ldots, t_{n}^{ \pm 1}\right]$. Let $k\left[q_{1}, \ldots, q_{n}, p_{1}, \ldots, p_{n}\right]$ be the $n$th Weyl algebra with generators $q_{i}, p_{j}$ satisfying $\left[p_{i}, q_{j}\right]=\delta_{i j}$. We regard this as the ring of differential operators on $k\left[t_{1}, \ldots, t_{n}\right]$ where $q_{i}$ acts as multiplication by $t_{i}$ and $p_{j}$ as $\partial / \partial t_{j}$. Let $B_{\mathbf{Q}}=\mathbf{Q}\left[q_{1} p_{1}, \ldots, q_{n} p_{n}\right]$ and $B=B_{\mathbf{Q}} \otimes_{\mathbf{Q}} k$. We may identify $B_{\mathbf{Q}}$ with the symmetric algebra on $\left(\mathbf{Q}^{n}\right)^{*}$. The next lemma will be used to find the Zariski closures of certain sets.

Lemma. Let $\Lambda$ be a subsemigroup of $\mathbf{Q}^{n}$ with $\mathbf{Q} \Lambda=\mathbf{Q}^{n}$. For $g \in\left(\mathbf{Q}^{n}\right)^{*}$ and $b \in \mathbf{Q}$ set $\Lambda_{b}=\{\lambda \in \Lambda \mid g(\lambda)=b\}$. Suppose that

(i) $\Lambda_{b} \neq \phi$,

(ii) $\operatorname{dim} \mathbf{Q} \Lambda_{0}=n-1$.

Then $\bar{\Lambda}_{h}=\left\{\lambda \in \mathbf{Q}^{n} \mid g(\lambda)=b\right\}$.

Proof. It suffices to show that if $f \in B_{\mathbf{Q}}$ and $f\left(\Lambda_{b}\right)=0$, then $f(\lambda)=0$ for all $\lambda \in \mathbf{Q}^{n}$ such that $g(\lambda)=b$. 
We first prove the case $b=0$. Write $X_{1}=q_{1} p_{1}, \ldots, X_{n}=q_{n} p_{n}$ and introduce new indeterminates $Y_{1}, \ldots, Y_{n}, T$. Suppose

$$
f(X+T Y)=f\left(X_{1}+T Y_{1}, \ldots, X_{n}+T Y_{n}\right)=\sum_{j=0}^{m} T^{j} g_{j}(X, Y)
$$

where $g_{j}(X, Y) \in \mathbf{Q}\left[X_{1}, \ldots, X_{n}, Y_{1}, \ldots, Y_{n}\right]$.

For $\lambda, \mu \in \Lambda_{0}$ and $t$ a positive integer we have $0=f(\lambda+t \mu)=\sum_{j=0}^{m} t^{j} g_{j}(\lambda, \mu)$. An argument using Vandermonde determinants shows that $g_{j}(\lambda, \mu)=0$ for each $j$. Taking $\lambda=0, \mu \in \Lambda_{0}$, and $t \in \mathbf{Q}^{+}$we find that $f\left(\mathbf{Q}^{+} \Lambda_{0}\right)=0$ and so $g_{j}(\lambda, \mu)=0$ for $\lambda, \mu \in \mathbf{Q}^{+} \Lambda_{0}$. Then taking $t=-1$ we find that $f\left(\mathbf{Q} \Lambda_{0}\right)=0$. Since $\operatorname{dim} \mathbf{Q} \Lambda_{0}=$ $n-1$, this proves the case $b=0$.

For the general case, there exists $\mu \in \Lambda$ such that $g(\mu)=b$, by (i). Define $f^{*} \in B_{Q}$ by $f^{*}(\nu)=f(\nu+\mu)$. Then $f^{*}\left(\Lambda_{0}\right)=0$ so by the case $b=0, f^{*}\left(\mathbf{Q} \Lambda_{0}\right)=$ 0 . If now $\lambda \in \mathbf{Q}^{n}$ and $g(\lambda)=b$ then $g(\lambda-\mu)=0$. Therefore $f(\lambda)=f^{*}(\lambda-\mu)=$ 0 as required.

We can now describe the Zariski closed sets $\overline{\Omega(\lambda)}$ of Theorem 2.4 as a union of hyperplanes. For $\beta \in \Delta$ and $i \notin \beta$ define $\overline{\Omega_{\beta, i}(\lambda)}=\left\{\mu \in \mathbf{Q}^{s} \mid 0 \leqslant \mu_{i}<-\lambda_{i}, \mu_{i} \in\right.$ Z . Then using Lemma $2.5 \overline{\Omega(\lambda)}=\bigcup_{\beta \in \Delta} \bigcup_{i \notin \beta} \overline{\Omega_{\beta, i}(\lambda)}$. To describe ann ${ }_{A} \overline{\Omega(\lambda)}$, set $S_{\lambda, \beta, i}=\prod_{j=0}^{-\lambda_{i}}\left(Q_{i} P_{i}-j\right)$ if $\beta \in \Delta, i \notin \beta, \lambda_{i}<0$, and $S_{\lambda, \beta, i}=1$ otherwise. Then $\operatorname{ann}_{A} \overline{\Omega(\lambda)}=S_{\lambda} A$ where $S_{\lambda}=\prod_{\beta \in \Delta} \prod_{i \notin \beta} S_{\lambda, \beta, i}$.

2.6. We next give a description of the ring $D\left(k \Lambda_{\beta}\right)$ wehre $\Lambda_{\beta}$ is a semigroup of the form $\Lambda_{\beta}=\mathbf{Z}^{n} \cap \bigcap_{i \notin \beta} H_{i}$ and $\mathbf{Z} \Lambda_{\beta}=\mathbf{Z}^{n}$. We may assume that the bounding halfspaces of $\mathbf{Q}^{+} \Lambda_{\beta}$ are the $H_{i}$ with $i \notin \beta$. Then by Theorem 2.3, $D\left(k \Lambda_{\beta}\right)=$ $\oplus_{\lambda \in \mathbf{Z}^{n}} q^{\lambda}$ ann $_{B} \omega_{\beta}(\lambda)$, where $\omega_{\beta}(\lambda)=\left\{\mu \in \Lambda_{\beta} \mid \mu+\lambda \notin \Lambda_{\beta}\right\}$. It is easily seen that $\omega_{\beta}(\lambda)=\bigcup_{i \notin \beta} \omega_{\beta, i}(\lambda)$ where $\omega_{\beta, i}(\lambda)=\left\{\mu \in \Lambda_{\beta} \mid f_{i}(\lambda+\mu)<0\right\}$.

The next lemma shows that $\overline{\omega_{\beta, i}(\lambda)}$ is a finite union of hyperplanes parallel to $H_{i}^{0}$.

Lemma. Suppose $i \notin \beta, H_{i}$ is a bounding halfspace of $\mathbf{Q} \Lambda_{\beta}$, and $f_{i}(\lambda)<0$. Then

$$
\overline{\omega_{\beta, i}(\lambda)}=\left\{\mu \in \mathbf{Q}^{n} \mid f_{i}(\mu) \in f_{i}\left(\mathbf{Z}^{n}\right) \text { and } 0 \leqslant f_{i}(\mu)<-f_{i}(\lambda)\right\} \text {. }
$$

Proof. The left side is contained in the right since $f_{i}$ is linear and the right side is Zariski closed in $\mathbf{Q}^{n}$.

We apply Lemma 2.5 with $g=f_{i}$. Suppose $f_{i}(\mu)=b \in f_{i}\left(\mathbf{Z}^{n}\right)$ and $0 \leqslant f_{i}(\mu)<$ $-f_{i}(\lambda)$. By Lemma 1.7, there is a $\nu \in \Lambda_{\beta}$ such that $f_{i}(\nu)=b$, so condition (i) of Lemma 2.5 holds. Since $H_{i}^{0}$ is a bounding hyperplane of $\mathbf{Q} \Lambda_{\beta}$, condition (ii) holds. We conclude that $\mu \in \overline{\omega_{\beta, i}(\lambda)}$.

2.7. By taking intersections over all $\beta \in \Delta$, we can obtain the following description of $D\left(Y_{\Delta} / G\right)$.

THEOREM. Suppose that for all $\beta \in \Delta, \mathbf{Z} \Lambda_{\beta}=\mathbf{Z}^{n}$ and that for $i \notin \beta, H_{i}$ is a bounding halfspace of $\mathbf{Q}^{+} \Lambda_{\beta}$. Then

$$
D\left(Y_{\Delta} / G\right)=\bigoplus_{\mu \in \mathbf{Z}^{n}} D\left(Y_{\Delta} / G\right)(\mu)
$$

where $D\left(Y_{\Delta} / G\right)(\mu)=q^{\lambda} \operatorname{ann}_{B} \overline{\omega(\lambda)}$, and $\overline{\omega(\lambda)}=\bigcup_{\beta \in \Delta} \bigcup_{i \notin \beta} \overline{\omega_{\beta, i}(\lambda)}$, and $\overline{\omega_{\beta, i}(\lambda)}$ is a union of hyperplanes as described in Lemma 2.6. 
2.8. We next investigate the map $\theta: D(Y)^{G} \rightarrow D\left(Y_{\Delta} / G\right)$. Let $I$ be the ideal of functions of $A$ which vanish on $\mathbf{Q} \Lambda$. Then $I$ is generated by linear polynomials, that is by first order homogeneous differential operators.

Lemma. Assume $Q^{\lambda} g \in D\left(U_{\beta}\right)^{G}$ where $\lambda \in \mathbf{Z}^{s} \cap V, g \in A$. Then

(i) $Q^{\lambda} g \in \operatorname{Ker} \theta_{\beta}$ if and only if $g \in I$,

(ii) if $\lambda \notin \mathbf{Z} \Lambda$ then $Q^{\lambda} g \in \operatorname{Ker} \theta_{\beta}$.

Proof. (i) $Q^{\lambda} g \in \operatorname{Ker} \theta_{\beta}$ if and only if for all $\mu \in \Lambda_{\beta}, 0=Q^{\lambda} g \cdot T^{\mu}=g(\mu) T^{\lambda+\mu}$. This occurs if and only if $g\left(\Lambda_{\beta}\right)=0$. By an argument used in the proof of Lemma 2.5 this is equivalent to $g(\mathbf{Q} \Lambda)=0$. Hence $Q^{\lambda} g \in \operatorname{Ker} \theta_{\beta}$ if and only if $g \in I$.

(ii) If $\lambda \notin \mathbf{Z} \Lambda$ and $\mu \in \Lambda_{\beta}$ then $\lambda+\mu \notin \Lambda_{\beta}$. Therefore $g\left(\Lambda_{\beta}\right)=0$ and $Q^{\lambda} g \in$ $\operatorname{Ker} \theta_{\beta}$ as in (i).

If $Q^{\lambda} g \in D\left(U_{\beta}\right)^{G}$, and $\lambda \in \mathbf{Z} \Lambda$, then the unique extension of $\theta_{\beta}\left(Q^{\lambda} g\right)$ to a differential operator on $k \mathbf{Z} \Lambda$ is given by

$$
\theta_{\beta}\left(Q^{\lambda} g\right) t^{\phi(\mu)}=g(\mu) t^{\phi(\lambda+\mu)} \quad \text { for } \mu \in \mathbf{Z} \Lambda .
$$

Here $\phi: \mathbf{Z} \Lambda \rightarrow \mathbf{Z}^{n}$ is the isomorphism of Proposition 1.3. It follows that if $x \in D\left(U_{\beta}\right)^{G} \cap D\left(U_{\gamma}\right)^{G}$ then $\theta_{\beta}(x)=\theta_{\gamma}(x)$ as elements of $D(k \mathbf{Z} \Lambda)$. The map $\theta$ : $D(Y)^{G}=\bigcap D\left(U_{\beta}\right)^{G} \rightarrow \cap D\left(\mathcal{O}\left(U_{\beta}\right)^{G}\right)=D\left(Y_{\Delta} / G\right)$ is obtained as the restriction of any $\theta_{\beta}, \beta \in \Delta$.

Proof of Theorem B. The second part of the theorem follows from Lemmas 2.2 and 2.8 since $\operatorname{Ker} \theta=D(Y)^{G} \cap \operatorname{Ker} \theta_{\beta}$.

Using Corollary 1.6 the action of $G$ on $\mathcal{O}\left(U_{\mathrm{s}}\right)$ may be extended to an action of a larger torus $G^{\prime}$ such that $\mathcal{O}\left(U_{\beta}\right)^{G^{\prime}}=\mathcal{O}\left(U_{\beta}\right)^{G}=k \Lambda_{\beta}$ for all $\beta \in \Delta$, and $\mathcal{O}\left(U_{\mathrm{s}}\right)^{G^{\prime}}=$ $k \mathbf{Z} \Lambda$. By Theorem 2.4 we have $D(Y)^{G^{\prime}}=\bigoplus_{\lambda \in \mathbf{Z} \Lambda} D(Y)^{G^{\prime}}(\lambda)$ where $D(Y)^{G^{\prime}}(\lambda)=$ $D(Y)^{G}(\lambda)$ for $\lambda \in \mathbf{Z} \Lambda$. By Lemma $2.8 \theta\left(D(Y)^{G}(\lambda)\right)=0$ unless $\lambda \in \mathbf{Z} \Lambda$. Hence in studying surjectivity we may replace $D(Y)^{G}$ with the subring $D(Y)^{G^{\prime}}$. In other words we can replace $G$ by $G^{\prime}$ and assume $\mathbf{Z} \Lambda_{\beta}=\mathbf{Z}^{s} \cap V$ for all $\beta \in \Delta$.

Let $\bar{\theta}: D\left(U_{\mathrm{s}}\right)^{G} \rightarrow D\left(\mathcal{O}\left(U_{\mathrm{s}}\right)^{G}\right)$ be the map obtained from restriction of the differential operators. For any $x \in D\left(U_{\beta}\right)^{G}, \bar{\theta}(x) \in D\left(\mathcal{O}\left(U_{\mathrm{s}}\right)^{G}\right)$ extends $\theta_{\beta}(x) \in D\left(\mathcal{O}\left(U_{\beta}\right)^{G}\right)$. The ring $D\left(U_{\mathbf{s}}\right)^{G}$ is a skew group ring of the group $M=\left\{Q^{\lambda} \mid \lambda \in \mathbf{Z} \Lambda\right\}$ over $B=k\left[Q_{1} P_{1}, \ldots, Q_{s} P_{s}\right]$. Therefore $\bar{\theta}$ is completely determined by its restriction to $M$ and $B$. We note that $\mathcal{O}\left(U_{\mathrm{s}}\right)^{G}$ is embedded in $D\left(U_{\mathrm{s}}\right)^{G}$ as the subring spanned by $\left\{Q^{\lambda} \mid \lambda \in \mathbf{Z} \Lambda\right\}$ and in $D\left(\mathcal{O}\left(U_{\mathbf{s}}\right)^{G}\right)$ as the subring $\left\{q^{\phi(\lambda)} \mid \lambda \in \mathbf{Z} \Lambda\right\}$. Hence, we have $\overline{\boldsymbol{\theta}}\left(Q^{\lambda}\right)=q^{\phi(\lambda)}$ for all $\lambda \in \mathbf{Z} \Lambda$. We claim the restriction of $\overline{\boldsymbol{\theta}}$ to $B$ is given by

$$
\bar{\theta}\left(Q_{i} P_{i}\right)=f_{i}\left(q_{1} p_{1}, \ldots, q_{n} p_{n}\right) \text { for } 1 \leqslant i \leqslant s .
$$

Indeed if $\lambda \in \mathbf{Z} \Lambda$ we have

$$
\begin{aligned}
\bar{\theta}\left(Q_{i} P_{i}\right) \cdot t^{\phi(\lambda)} & =\lambda_{i} t^{\phi(\lambda)}=\left(x_{i} \phi^{-1}\right)(\phi(\lambda)) t^{\phi(\lambda)} \\
& =\left(\psi^{*} x_{i}\right)(\phi(\lambda)) t^{\phi(\lambda)}=f_{i}(\phi(\lambda)) t^{\phi(\lambda)} \\
& =f_{i}\left(q_{1} p_{1}, \ldots, q_{n} p_{n}\right) \cdot t^{\phi(\lambda)} .
\end{aligned}
$$

We note that the restriction of $\theta$ to $A$ gives a surjective map from $A$ to $B$. To see this note that the linear map $\psi: \mathbf{Q}^{n} \rightarrow \mathbf{Q}^{s}$ has rank $n$, and so $\psi^{*}:\left(\mathbf{Q}^{s}\right)^{*} \rightarrow\left(\mathbf{Q}^{n}\right)^{*}$ has rank $n$ also. Hence the linear functionals $f_{i}=\psi^{*} x_{i}, 1 \leqslant i \leqslant s \operatorname{span}\left(\mathbf{Q}^{n}\right)^{*}$ and so the linear polynomials $f_{i}=\theta\left(Q_{i} P_{i}\right)$ generate $B$. 
To prove the theorem observe that $\theta: D(Y)^{G} \rightarrow D\left(Y_{\Delta} / G\right)$ will be surjective if and only if for all $\lambda \in \mathbf{Z} \Lambda$, the restriction of $\theta$ to $D(Y)^{G}(\lambda) \rightarrow D\left(Y_{\Delta} / G\right)(\phi(\lambda))$ is surjective. Using Theorems 2.4 and 2.7 we see that $\theta$ is surjective if and only if for all $\lambda \in \mathbf{Z} \Lambda$ we have $\theta\left(\operatorname{ann}_{A} \overline{\Omega(\lambda)}\right)=\operatorname{ann}_{B} \overline{\omega(\phi(\lambda))}$.

We first show that condition (a) is necessary for surjectivity. If (a) fails then for some $i, j \in \alpha, i \neq j$, we have $c f_{i}=f_{j}$ for some $c>0$. Choose $\lambda \in \mathbf{Z} \Lambda$ with $\lambda_{i}<0$. Then $\overline{\Omega(\lambda)}$ contains the hyperplanes $\mathscr{H}_{i}^{0}$ and $\mathscr{H}_{j}^{0}$. Therefore $f_{i}^{2} \in \theta\left(\operatorname{ann}_{A} \overline{\Omega(\lambda)}\right)$, but $f_{i} \notin \theta\left(\operatorname{ann}_{A} \overline{\Omega(\lambda)}\right)$. Therefore $\theta$ is not surjective, since $\operatorname{ann}_{B} \overline{\omega(\phi(\lambda))}$ is a semiprime ideal.

Henceforth we assume that (a) holds. We have

$$
\theta\left(\operatorname{ann}_{A} \overline{\Omega(\lambda)}\right)=\operatorname{ann}_{B}(\overline{\Omega(\lambda)} \cap \mathbf{Q} \Lambda),
$$

since now distinct hyperplanes in $\overline{\Omega(\lambda)}$ now have distinct intersections with $Q \Lambda$.

Since we always have $\theta\left(\operatorname{ann}_{A} \overline{\Omega(\lambda)}\right) \subset \operatorname{ann}_{B} \overline{\omega(\phi(\lambda))}$ it now suffices to show that conditions (b) and (c) hold if and only if for all $\lambda \in \mathbf{Z} \Lambda$ we have $\overline{\Omega(\lambda)} \cap Q \Lambda$ $\subset \overline{\omega(\phi(\lambda))}$.

Suppose that (b) and (c) hold. If $H$ is a hyperplane contained in $\overline{\Omega(\lambda)} \cap \mathbf{Q} \Lambda$, there exists $i \notin \bigcap_{\beta \in \Delta} \beta$ with $x_{i}(\lambda)<0$ and an integer $b$ with $0 \leqslant b<-x_{i}(\lambda)$ such that

$$
H=\left\{\mu \in \mathbf{Q} \Lambda \mid x_{j}(\mu) \geqslant 0 \text { for } j \notin \bigcap_{\beta \in \Delta} \beta \text { and } x_{i}(\mu)=b\right\} .
$$

We have $i \in \alpha$, so by (b) $\operatorname{ker} f_{i}=\operatorname{ker} x_{i} \cap \mathbf{Q} \Lambda$ is a bounding hyperplane of $\mathbf{Q} \Lambda_{\beta}$ for some $\beta \in \Delta$. Since $f_{i}(\mathbf{Z} \Lambda)=\mathbf{Z}$, we obtain $H \subset \overline{\omega_{\beta, i}(\phi(\lambda))} \subset \overline{\omega_{\beta}(\phi(\lambda))}$ as required.

Conversely, suppose that some induced hyperplane $\mathbf{Q} \Lambda \cap \operatorname{ker} x_{i}$ is not a bounding hyperplane of $\mathbf{Q}^{+} \Lambda_{\beta}$ for any $\beta \in \Delta$. Choose $\lambda \in \mathbf{Z} \Lambda$ with $f_{i}(\lambda)<0$. Then the hyperplane $\left\{\mu \in \mathbf{Q} \Lambda \mid f_{i}(\mu)=0\right\}$ is contained in $\overline{\Omega(\lambda)} \cap \mathbf{Q} \Lambda$ but not in $\overline{\omega_{\beta}(\phi(\lambda))}$ for any $\beta$. Hence condition (b) is necessary for surjectivity.

Finally assume conditions (a) and (b) hold, but that (c) fails. Then for some induced hyperplane $\mathbf{Q} \Lambda \cap \operatorname{ker} x_{i}$ of $\mathbf{Q}^{+} \Lambda_{\beta}, \beta \in \Delta$, we have $x_{i}(\mathbf{Z} \Lambda)=a \mathbf{Z}$ with $a>1$. Choose $\lambda \in \mathbf{Z} \Lambda$ with $f_{i}(\lambda)<0$. Then the hyperplane $\left\{\mu \in \mathbf{Q} \Lambda \mid f_{i}(\mu)=1\right\}$ is contained in $\overline{\Omega(\lambda)} \cap \mathbf{Q} \Lambda$ but not in $\overline{\omega_{\beta}(\phi(\lambda))}$ for any $\beta$. This shows conditions (a)-(c) are necessary for surjectivity and completes the proof of Theorem B.

2.9. Examples. We first give some examples to illustrate the independence of conditions (a)-(c) of Theorem B, part 1. We suppose that $G=\mathrm{GL}(1)^{r}$ acts on $\mathcal{O}\left(U_{\mathbf{s}}\right)=k\left[T_{1}{ }^{ \pm}, \ldots, T_{s}{ }^{ \pm 1}\right]$ by the rule $g \cdot T_{j}=\prod_{i} g_{i}^{a_{i j}} T_{j}$ where $\left(a_{i j}\right)$ is an $r \times s$ matrix with integer entries.

(a) Suppose $r=1, s=2,\left(a_{i j}\right)=(1-1)$, and $\Delta=\{\phi\}$. Then $T^{\lambda} \in \mathcal{O}\left(U_{\phi}\right)=$ $k\left[T_{1}, T_{2}\right]$ is $G$-invariant if and only if $\lambda \in \mathbf{Z}^{+}(1,1)=\Lambda$. Clearly the halfspaces in $\mathbf{Q}^{2}$ defined by $x_{1} \geqslant 0$ and $x_{2} \geqslant 0$ give the same induced halfspaces of $\mathbf{Q} \Lambda$. Hence condition (a) of Theorem B fails. It is easily verified that conditons (b) and (c) hold.

(b) Suppose $r=1, s=3,\left(a_{i j}\right)=\left(\begin{array}{lll}1 & 1 & -2\end{array}\right)$, and $\Delta=\{\phi\}$. Then $T^{\lambda} \in \mathcal{O}\left(U_{\phi}\right)$ $=k\left[T_{1}, T_{2}, T_{3}\right]$ is $G$-invariant if and only if $\lambda$ belongs to the semigroup $\Lambda$ generated by $(2,0,1),(0,2,1)$ and $(1,1,1)$. We note that the induced halfspace of $\mathbf{Q} \Lambda$ defined 
by $x_{3} \geqslant 0$ is not a bounding halfspace of $\mathbf{Q}^{+} \Lambda$. Therefore condition (b) of Theorem B fails. It is easily checked that conditions (a), (c) of Theorem B hold. Note that if we let $\Delta=\{\{3\}\}$ instead, then $\mathcal{O}\left(U_{\{3\}}\right)=k\left[T_{1}, T_{2}, T_{3}^{ \pm 1}\right]$ and the halfspace of $\mathbf{Q} \Lambda$ defined by $x_{3} \geqslant 0$ is no longer an induced halfspace. Hence conditions (a)-(c) of Theorem B hold in this case. Also we have $\mathcal{O}\left(U_{\phi}\right)^{G}=\mathcal{O}\left(U_{\{3\}}\right)^{G}$.

(c) Suppose $r=1, s=3,\left(a_{i j}\right)=\left(\begin{array}{lll}1 & 2 & -4\end{array}\right)$ and $\Delta=\{\{3\}\}$. Then $\mathcal{O}\left(U_{\{3\}}\right)^{G}=$ $k \Lambda$ where $\Lambda$ is the semigroup generated by $(4,0,1),(2,1,1)$, and $(0,2,1)$. Conditions (a), (b) of Theorem B hold, but $x_{1} \geqslant 0$ defines an induced halfspace of $\mathbf{Q}^{+} \Lambda$ and $x_{1}(\mathbf{Z} \Lambda)=2 \mathbf{Z}$. Note also that $G$ satisfies the dimension equality and contains the pseudoreflection $(-1,1,1)$ which fixes the hyperplane defined by $x_{1}=0$.

(d) Finally, we give an example to show that the definition of $D\left(Y_{\Delta} / G\right)$ depends on $\Delta$. Let $r=1, s=3,\left(a_{i j}\right)=\left(\begin{array}{lll}1 & 1 & -2\end{array}\right)$. We have seen in (b) that $\mathcal{O}\left(U_{\phi}\right)^{G}=$ $\mathcal{O}\left(U_{\{3\}}\right)^{G}$ and the map $D\left(\mathcal{O}\left(U_{\{3\}}\right)\right)^{G} \rightarrow D\left(\mathcal{O}\left(U_{\phi}\right)^{G}\right)$ is surjective. Hence $P_{1} P_{2} Q_{3}^{-1} \in$ $D\left(\mathcal{O}\left(U_{\{3\}}\right)\right)^{G}$ induces a differential operator on $\mathcal{O}\left(U_{\phi}\right)^{G}$. On the other hand we have $\mathcal{O}\left(U_{\{1\}}\right)^{G}=k\left[T_{1}^{-1} T_{2}, T_{1}^{2} T_{3}\right]$ and $P_{1} P_{2} Q_{3}^{-1} \cdot T_{1}^{-1} T_{2}=-T_{1}^{-2} T_{3}^{-1} \notin \mathcal{O}\left(U_{\{1\}}\right)^{G}$. Hence $D\left(\mathcal{O}\left(U_{\phi}\right)^{G}\right) \nsubseteq D\left(\mathcal{O}\left(U_{\{1\}}\right)^{G}\right)$. Now we can write $U_{\phi}=U_{\phi} \cup U_{\{1\}}$. Thus with $\Delta=\{\phi\}$ and $\Delta^{\prime}=\{\phi,\{1\}\}$, we have $Y=\cup_{\beta \in \Delta} U_{\beta}=\bigcup_{\beta \in \Delta^{\prime}} U_{\beta}$ and $D\left(Y_{\Delta} / G\right) \nsubseteq D\left(Y_{\Delta^{\prime}} / G\right)$.

\section{Centers.}

3.1. We denote the center of a ring $R$ by $Z(R)$, and the Ore quotient ring of $R$ by $Q(R)$ when it exists. Let $R$ be a subring of $D\left(U_{\mathrm{s}}\right)=k\left[Q_{1}^{ \pm 1}, \ldots, Q_{s}^{ \pm 1}, P_{1}, \ldots, P_{s}\right]$ containing $A=k\left[Q_{1} P_{1}, \ldots, Q_{s} P_{s}\right]$. By Lemma 2.2, $R=\oplus_{\lambda \in \mathbf{Z}^{s}} R(\lambda)$ where $R(\lambda)$ $=\left\{x \in R \mid\left[Q_{i} P_{i}, x\right]=\lambda_{i} x\right.$ for $\left.1 \leqslant i \leqslant s\right\}$. Let $\Lambda=\left\{\lambda \in \mathbf{Z}^{s} \mid R(\lambda) \neq 0\right\}$, and $S=$ $k\left[Q_{1} P_{1}, \ldots, Q_{s} P_{s}, Q^{\lambda} ; \lambda \in \mathbf{Z} \Lambda\right]$. We denote by $\mathrm{GK}(R)$ the Gelfand-Kirillov dimension of $R$; see [KL].

LEMMA. With the above notation $\mathrm{GK}(R)=s+\operatorname{rank}(\mathbf{Z} \Lambda)$.

Proof. We may assume that $R$ is finitely generated as a $k$-algebra. Since $R \subset S$, we have $\mathrm{GK}(R) \leqslant \mathrm{GK}(S)$. A straightforward calculation shows that $\operatorname{GK}(S)=s+$ $\operatorname{rank}(\mathbf{Z} \Lambda)$.

Suppose $\lambda_{1}, \ldots, \lambda_{r}$ are $\mathbf{Z}$-linearly independent elements of $\Lambda$, where $r=$ $\operatorname{rank}(\mathbf{Z} \Lambda)$. Choose a finite-dimensional subspace $W$ of $R$ containing $1, Q_{1} P_{1}, \ldots, Q_{s} P_{s}$ and such that $W \cap R\left(\lambda_{i}\right) \neq 0$ for $i=1, \ldots, r$. Let $R_{m}(\lambda)=W^{m} \cap R(\lambda)$. For $\lambda=\sum a_{i} \lambda_{i} \in \sum \mathbf{Z}_{+} \lambda_{i}$ define $|\lambda|=\sum a_{i}$. Clearly $R_{|\lambda|}(\lambda) \neq 0$ for all $\lambda \in \sum \mathbf{Z}_{+} \lambda_{i}$. Since the number of monomials of degree at most $d$ in $s$ indeterminates is $\left(\begin{array}{c}s+d \\ s\end{array}\right)$ we see that $\operatorname{dim} R_{m}(\lambda) \geqslant\left(\begin{array}{c}s+m-|\lambda| \\ s\end{array}\right)$, for $m \geqslant|\lambda|$. Therefore $\operatorname{dim} W^{m} \geqslant \sum\left(\begin{array}{c}s+m-|\lambda| \\ s\end{array}\right)$, where the sum is over all $\lambda \in \sum \mathbf{Z}_{+} \lambda_{i}$ with $|\lambda| \leqslant m$. By induction on $r$ it is possible to show that the right side is bounded below by a polynomial in $m$ of degree $s+r$. Hence $\mathrm{GK}(R) \geqslant s+r$ and this proves the lemma.

3.2. Corollary. (i) Suppose $G=\mathrm{GL}(1)^{r}$ acts faithfully on $k^{s}$. Then $\operatorname{GK}\left(D(Y)^{G}\right)$ $=2 s-r$.

(ii) Let $Y=\cup U_{\beta}$ and suppose the common quotient field of the rings $\mathcal{O}\left(U_{\beta}\right)^{G}$ has transcendence degree $n$ over $k$. Then $\operatorname{GK}\left(D\left(Y_{\lrcorner} / G\right)\right)=2 n$. 
Proof. This follows immediately from Lemma 3.1, and Theorems 2.4 and 2.7. It follows from [KL, Theorem 4.12(a)] and Lemma 3.1 that $Q(R)$ exists.

3.3. LeMma. With the above notation $Q(R)=Q(S)$.

Proof. Since $R \subset S$, and $S$ is a domain, it suffices to show $S \subset Q(R)$. If $\lambda \in \Lambda$, there exists $\alpha \in A, \alpha \neq 0$ such that $Q^{\lambda} \alpha \in R$. Since $A \subset R$, it follows that $Q^{\lambda} \in$ $Q(R)$. Hence $Q^{\lambda} \in Q(R)$ for $\lambda \in \mathbf{Z} \Lambda$ and $S \subset Q(R)$.

Now suppose $g_{1}, \ldots, g_{r}$ form a basis for the kernel of the restriction map $\left(\mathbf{Q}^{s}\right)^{*} \rightarrow(\mathbf{Q} \Lambda)^{*}$, and choose $h_{1}, \ldots, h_{l} \in\left(\mathbf{Q}^{s}\right)^{*}, t=s-r$ so that $g_{1}, \ldots, g_{r}$, $h_{1}, \ldots, h_{t}$ form a basis of $\left(\mathbf{Q}^{s}\right)^{*}$. We regard the elements $g_{j}$ and $h_{i}$ as elements of $A=k\left[Q_{1} P_{1}, \ldots, Q_{s} P_{s}\right]$. Then $A$ is a polynomial ring in $h_{1}, \ldots, h_{t}$ over $C=$ $k\left[g_{1}, \ldots, g_{r}\right]$.

THEOREM C. With the above notation $Z(R)=C$.

Proof. We show first that $C \subseteq Z(S)$. Since $S$ is the twisted group algebra of $\left\{Q^{\lambda} \mid \lambda \in \mathbf{Z} \Lambda\right\}$ over $A$, it is enough to show that $\left[Q^{\lambda}, g_{j}\right]=0$ for $\lambda \in \mathbf{Z} \Lambda$ and $1 \leqslant j \leqslant r$. Now

$$
\begin{aligned}
{\left[Q^{\lambda}, g_{j}\right] } & =Q^{\lambda}\left\{g_{j}\left(Q_{1} P_{1}, \ldots, Q_{s} P_{s}\right)-g_{j}\left(Q_{1} P_{1}+\lambda_{1}, \ldots, Q_{s} P_{s}+\lambda_{s}\right)\right\} \\
& =Q^{\lambda}\left\{g_{j}\left(Q_{1} P_{1}, \ldots, Q_{s} P_{s}\right)-g_{j}\left(Q_{1} P_{1}, \ldots, Q_{s} P_{s}\right)-g_{j}(\lambda)\right\}=0,
\end{aligned}
$$

as $g_{j}$ is a linear polynomial and $g_{j}(\lambda)=0$.

Since $C \subseteq R \subseteq S$, it is now sufficient to prove that $Z(R) \subseteq C$. Note that if $x \in Z(R)$, then $[\xi, x]=0$ for all $\xi \in \operatorname{Lie} \Gamma=k Q_{1} P_{1}+\cdots+k Q_{s} P_{s}$. Hence $x \in$ $R(0)=A$.

We let $\leqslant$ denote the natural partial order on $\left(\mathbf{Z}_{+}\right)^{t}$, that is for $\alpha, \beta \in\left(\mathbf{Z}_{+}\right)^{t}$, $\alpha \leqslant \beta$ if and only if $\alpha_{i} \leqslant \beta_{i}$ for $1 \leqslant i \leqslant t$.

Suppose $x=\sum_{\beta \in\left(\mathbf{Z}_{+}\right)^{\prime}} z_{\beta} h^{\beta} \in A$ is central in $R$ where $z_{\beta} \in C$. If $x \notin C$, then $z_{\beta} \neq 0$ for some $\beta>0$. We choose an element $\alpha \in\left(\mathbf{Z}_{+}\right)^{t}$ maximal under the partial order $\leqslant$ such that $z_{\alpha} \neq 0$. Suppose $\alpha_{i} \neq 0$. Since the restrictions of $h_{1}, \ldots, h_{t}$ form a basis for $(\mathbf{Q} \Lambda)^{*}$, there exists $\lambda \in \mathbf{Z} \Lambda$ such that $h_{j}(\lambda)=0$ if $j \neq i$ and $h_{i}(\lambda) \neq 0$. Since $x \in Z(R)$ and $S \subset Q(R)$ we have

$$
0=\left[x, Q^{\lambda}\right]=Q^{\lambda}\left\{\sum_{\beta} z_{\beta}\left(h^{\beta}\left(Q_{1} P_{1}+\lambda_{1}, \ldots, Q_{s} P_{s}+\lambda_{s}\right)-h^{\beta}\left(Q_{1} P_{1}, \ldots, Q_{s} P_{s}\right)\right)\right\} .
$$

For any $\beta$ we have

$$
\begin{aligned}
h^{\beta}\left(Q_{1} P_{1}+\lambda_{1}, \ldots,\right. & \left.Q_{s} P_{s}+\lambda_{s}\right)-h^{\beta}\left(Q_{1} P_{1}, \ldots, Q_{s} P_{s}\right) \\
& =\beta_{i} h_{i}(\lambda) h^{\beta^{\prime}}\left(Q_{1} P_{1}, \ldots, Q_{s} P_{s}\right)+\text { lower degree terms }
\end{aligned}
$$

where $\beta^{\prime}=\left(\beta_{1}, \ldots, \beta_{i-1}, \beta_{i}-1, \beta_{i+1}, \ldots, \beta_{t}\right)$.

For $\beta \neq \alpha$, none of the above terms involves $h^{\alpha^{\prime}}$ and it follows that the coefficient of $h^{\alpha^{\prime}}$ in the above sum is $\alpha_{i} h_{i}(\lambda) z_{\alpha} \neq 0$. This contradiction shows that $Z(R) \subseteq C$ which proves the theorem. 
3.4. We now specialize Theorem $\mathrm{C}$ to the rings $D(Y)^{G}$ and $D\left(Y_{\Delta} / G\right)$. The fixed ring $\mathcal{O}\left(U_{s}\right)^{G}$ is the group algebra of $\Lambda_{\mathrm{s}}=\left\{\lambda \in \mathbf{Z}^{s} \mid m_{i}(\lambda)=0\right\}$ where the $m_{i} \in$ $\left(\mathbf{Q}^{s}\right)^{*}$ are given by equation (2) of $\S 1$. In the notation of Theorem $2.4, \Lambda_{\mathrm{s}}=\mathbf{Z}^{s} \cap V$. This gives the first part of the following corollary.

COROLlaRY. With the above notation

(i) $Z\left(D(Y)^{G}\right)=k\left[m_{1}, \ldots, m_{r}\right]$.

(ii) $Z\left(D\left(Y_{\Delta} / G\right)\right)=k$.

Proof. Part (ii) follows from Theorem $\mathrm{C}$ and Theorem 2.7.

3.5. Recall that for $\lambda \in \mathbf{Z}^{s} \cap V$, ann $\bar{\Omega} \overline{\Omega(\lambda)}=S_{\lambda} A$, where $S_{\lambda}$ is defined after Lemma 2.5 .

Lemma. Assume $G$ satisfies the dimension equality on $\mathcal{O}\left(U_{\beta}\right)$ for $\beta \in \Delta$. For $1 \leqslant l \leqslant r$, let $I_{l}=\sum_{k=1}^{l} m_{k} A$. Then $S_{\lambda} A \cap I_{l}=S_{\lambda} I_{l}$.

Proof. If $Q_{i} P_{i}-j$ is a factor of $S_{\lambda}$ then for some $\beta \in \Delta$ and $i \notin \beta$ we have $\lambda_{i}<0$ and $0 \leqslant j<-\lambda_{i}$. Since $\lambda \in Q \Lambda$ and $x_{i}(\lambda)=\lambda_{i}$ we have $Q \Lambda \nsubseteq \operatorname{ker} x_{i}$, and clearly $Q \Lambda \nsubseteq \operatorname{ker}\left(x_{i}-j\right)$ if $j \neq 0$. Hence $\left(Q_{i} P_{i}-j\right) \notin I_{r}$ which is the ideal of functions of $A$ that vanish on $\mathbf{Q} \Lambda=V$. Therefore $\left(Q_{i} P_{i}-j\right) \notin I_{l}$. Since $I_{l}$ is a prime ideal of $A$ it follows that $S_{\lambda} \notin I_{l}$ and $S_{\lambda} A \cap I_{l}=S_{\lambda} I_{l}$.

3.6. Note that if $G$ satisfies the dimension equality on $\mathcal{O}\left(U_{\beta}\right), \beta \in \Delta$, then $I_{r}=I$ is the ideal used in the proof of Theorem B. We can now reformulate Theorem B in this case.

Corollary. Assume $G$ satisfies the dimension equality on $\mathcal{O}\left(U_{\beta}\right), \beta \in \Delta$. Let $\theta$ : $D(Y)^{G} \rightarrow D\left(Y_{\Delta} / G\right)$ be the natural map.

(1) $\theta$ is surjective if and only if

(a) The induced halfspaces of $\mathbf{Q} \Lambda$ are distinct and are precisely the bounding halfspaces of the convex cones $\mathbf{Q}^{+} \Lambda_{\beta}, \beta \in \Delta$.

(b) If $\mathbf{Q} \Lambda \cap \mathscr{H}_{j}$ is a bounding halfspace of $\mathbf{Q}^{+} \Lambda_{\beta}$, then $G$ contains no nonidentity pseudoreflection fixing the hyperplane defined by $x_{j}=0$.

(2) $\operatorname{Ker} \theta=D(Y)^{G} I=D(Y)^{G}\left(\operatorname{Ker} \theta \cap Z\left(D(Y)^{G}\right)\right)$.

Proof. (1) follows immediately from Theorem B and Lemma 1.9.

(2) Theorem B gives $\operatorname{Ker} \theta=D\left(U_{\mathrm{s}}\right)^{G} I \cap D(Y)^{G}$. In particular $I \subseteq \operatorname{Ker} \theta$. Also $I \subseteq Z\left(D(Y)^{G}\right)$ by Corollary 3.4. If $g \in A$ and $Q^{\lambda} g \in(\operatorname{Ker} \theta)(\lambda)$, we have $g \in S_{\lambda} A$ $\cap I=S_{\lambda} I$. Therefore $Q^{\lambda} g \in Q^{\lambda} S_{\lambda} I \subseteq D(Y)^{G} I$. Together with Lemma 2.2 this proves (2).

3.7. In later applications we shall often require the conditions of the corollary to be sastisfied. It is important to know that we can do this without changing the ring $D\left(Y_{\Delta} / G\right)$ up to isomorphism.

Proposition. Suppose the torus $G$ acts on $Y=\cup_{\beta \in \Delta} U_{\beta}$. Then there is a set of subsets $\Sigma$ of $\mathbf{s}$ such that if $X=\cup_{\beta \in \Sigma} U_{\beta}$, then there is an action of a possibly larger torus $G^{\prime}$ on $X$ such that

(i) $D\left(X_{\Sigma} / G^{\prime}\right) \cong D\left(Y_{\Delta} / G\right)$.

(ii) $G^{\prime}$ satisfies the dimension equality on $\mathcal{O}\left(U_{\beta}\right), \beta \in \Sigma$.

(iii) The map $\theta: D(X)^{G} \rightarrow D\left(X_{\Sigma} / G^{\prime}\right)$ is surjective. 
Proof. We define an equivalence relation $\sim$ on $\alpha=\alpha(\Delta)$ by $i \sim j$ for $i, j \in \alpha$ if and only if $f_{i}=a f_{j}$ for some $a>0$. Let $\gamma$ be a set of representatives for the equivalence classes of $\sim$. For $\beta \in \Delta$, we can choose a set $\beta^{\prime}$ containing $\beta$ such that the bounding halfspaces of $\mathbf{Q}^{+} \Lambda_{\beta}=\mathbf{Q}^{+} \Lambda_{\beta^{\prime}}$ have the form $\left\{\mathbf{Q} \Lambda \cap \mathscr{H}_{j} \mid j \in \mathbf{s} \backslash \beta^{\prime}\right\}$ and $\left(\mathbf{s} \backslash \beta^{\prime}\right) \subseteq \gamma$. Let $\Sigma=\left\{\beta^{\prime} \mid \beta \in \Delta\right\}$. If $X=\bigcup_{\beta^{\prime} \in \Sigma} U_{\beta^{\prime}}$, then $G$ acts on $X$, conditions 1(a) and (b) of Theorem B now hold, and we have $\mathcal{O}\left(U_{\beta^{\prime}}\right)^{G}=\mathcal{O}\left(U_{\beta}\right)^{G}$ for all $\beta$. By embedding $G$ in a larger torus, we may assume the dimension equality holds on $\mathcal{O}\left(U_{\beta}\right)$ for all $\beta \in \Sigma$. Since these reductions do not change the fixed rings we have $\bigcap_{\beta \in \Delta} D\left(\mathcal{O}\left(U_{\beta}\right)^{G}\right)=\bigcap_{\beta^{\prime} \in \Sigma} D\left(\mathcal{O}\left(U_{\beta^{\prime}}\right)^{G^{\prime}}\right)$.

To obtain condition 1(c) of Theorem B, we apply Lemma 1.8. Since we replace $\mathcal{O}\left(U_{\beta^{\prime}}\right)^{G^{\prime}}$ by an isomorphic ring some care must be taken. Suppose $\phi: \mathbf{Q}^{s} \rightarrow \mathbf{Q}^{s}$ is the isomorphism of Lemma 1.8. We obtain an isomorphism, also denoted by $\phi$ from $k \mathbf{Z} \Lambda$ to $k \phi \mathbf{Z} \Lambda$. The map $\phi^{*}: D(k \mathbf{Z} \Lambda) \rightarrow D(k \phi \mathbf{Z} \Lambda)$ defined by $\left(\phi^{*} d\right)(f)=$ $\phi\left(d\left(\phi^{-1} f\right)\right)$ is an isomorphism, and using Lemma 2.1, we can show that $\phi^{*}$ maps $\bigcap_{\beta^{\prime} \in \Sigma} D\left(k \Lambda_{\beta^{\prime}}\right)$ onto $\bigcap_{\beta^{\prime} \in \Sigma} D\left(k \phi \Lambda_{\beta^{\prime}}\right)$.

\section{Graded rings.}

4.1. We filter the rings $D(Y)^{G}$ and $D\left(Y_{\Delta} / G\right)$ by the order of the differential operators. In $D\left(U_{\mathrm{s}}\right)$ we let $\mathscr{E}_{t}$ denote the span of all differential operators of order at most $t$, and $\mathscr{D}_{t}=\mathscr{E}_{t} \cap D(Y)$. Then $\mathscr{E}_{t}$ is the free $\mathcal{O}\left(U_{\mathrm{s}}\right)$-module generated by monomials in $Q_{1} P_{1}, \ldots, Q_{s} P_{s}$ of degree at most $t$. We also set $\mathscr{D}_{-1}=\mathscr{E}_{-1}=0$. Then

$$
\operatorname{Gr} D(Y)=\bigoplus_{t \geqslant 0} \frac{\mathscr{D}_{t}}{\mathscr{D}_{t-1}} \subseteq \operatorname{Gr} D\left(U_{\mathrm{s}}\right)=\bigoplus \frac{\mathscr{E}_{t}}{\mathscr{E}_{t-1}}
$$

The action of $G$ preserves the filtrations $\left\{\mathscr{D}_{t}\right\}$ and $\left\{\mathscr{E}_{t}\right\}$, and hence $G$ acts on $\operatorname{Gr} D(Y)$ by

$$
g \cdot\left(a+\mathscr{D}_{t-1}\right)=\left(g \cdot a+\mathscr{D}_{t-1}\right) \quad \text { for } a \in \mathscr{D}_{t} .
$$

We may filter $D(Y)^{G}$ by the subspaces $\mathscr{D}_{t} \cap D(Y)^{G}$, and it follows that

$$
\begin{aligned}
\operatorname{Gr}\left(D(Y)^{G}\right) & =\bigoplus_{t \geqslant 0} \frac{D(Y)^{G} \cap \mathscr{D}_{t}}{D(Y)^{G} \cap \mathscr{D}_{t-1}} \cong \bigoplus_{t \geqslant 0} \frac{\left(D(Y)^{G} \cap \mathscr{D}_{t}\right)+\mathscr{D}_{t-1}}{\mathscr{D}_{t-1}} \\
& =(\operatorname{Gr} D(Y))^{G} .
\end{aligned}
$$

In the ring $D(k \mathbf{Z} \Lambda)=k\left[q_{1}^{ \pm 1}, \ldots, q_{n}^{ \pm 1}, p_{1}, \ldots, p_{n}\right]$ we let $E_{t}$ denote the span of all differential operators of order at most $t$, and $D_{t}=E_{t} \cap D\left(Y_{\Delta} / G\right)$. Then $E_{t}$ is the free $k \mathbf{Z} \Lambda$-module generated by monomials in $q_{1} p_{1}, \ldots, q_{n} p_{n}$ of degree at most $t$. As before

$$
\operatorname{Gr}\left(D\left(Y_{\Delta} / G\right)\right)=\bigoplus D_{t} / D_{t-1} \subseteq \bigoplus E_{t} / E_{t-1}=\operatorname{Gr}(D(k \mathbf{Z} \Lambda)) .
$$

It follows from the proof of Theorem B that the map $\theta: D(Y)^{G} \rightarrow D\left(Y_{\Delta} / G\right)$ preserves the filtrations defined above, that is $\theta\left(\mathscr{D}_{t} \cap D(Y)^{G}\right) \subseteq D_{t}$ for all $t \geqslant 0$. Hence there is an induced map $\operatorname{Gr} \theta: \operatorname{Gr}\left(D(Y)^{G}\right) \rightarrow \operatorname{Gr}\left(D\left(Y_{\Delta} / G\right)\right)$ defined by $\operatorname{Gr} \theta\left(a+\left(\mathscr{D}_{t-1} \cap D(Y)^{G}\right)\right)=\theta(a)+D_{t-1}$ for $a \in \mathscr{D}_{t} \cap D(Y)^{G}$. It is routine to 
check that $\operatorname{Gr} \theta$ is surjective if and only if $\theta$ is surjective and that the diagram

$$
\begin{array}{ccc}
D(Y)^{G} & \stackrel{\theta}{\rightarrow} & D\left(Y_{\Delta} / G\right) \\
\Pi_{1} \downarrow & & \downarrow \Pi_{2} \\
\operatorname{Gr} D(Y)^{G} & \stackrel{\operatorname{Gr} \theta}{\rightarrow} & \mathrm{Gr} D\left(Y_{\Delta} / G\right)
\end{array}
$$

commutes. Here if $a \in\left(\mathscr{D}_{t} \cap D(Y)^{G}\right) \backslash\left(\mathscr{D}_{t-1} \cap D(Y)^{G}\right)$ we define $\Pi_{1}(a)=a+$ $\left(\mathscr{D}_{t-1} \cap D(Y)^{G}\right)$. The map $\Pi_{2}$ is defined similarly.

TheOREM. The graded rings $\operatorname{Gr}\left(D(Y)^{G}\right)=(\operatorname{Gr} D(Y))^{G}$ and $\operatorname{Gr}\left(D\left(Y_{\Delta} / G\right)\right)$ are finitely generated commutative $k$-algebras.

Proof. The graded ring of $\operatorname{Gr} D(Y) \cong k\left[X_{1}, \ldots, X_{s}, Y_{1}, \ldots, Y_{s}, X_{i}^{-1} ; i \in \cap_{\beta \in \Delta} \beta\right]$ is a localization of a commutative polynomial ring at some of the indeterminates, and $G=\mathrm{GL}(1)^{r}$ acts linearly on the subspace spanned by $X_{1}, \ldots, X_{s}, Y_{1}, \ldots, Y_{s}$. A well-known result from invariant theory, [Hu, \$14, Exercise 2] implies that the fixed ring $(\mathrm{Gr} D(Y))^{G}$ is finitely generated as a $k$-algebra.

To show that $\operatorname{Gr} D\left(Y_{\Delta} / G\right)$ is finitely generated, we may assume the map $\theta$ is surjective by Proposition 3.7. Hence $\mathrm{Gr} \theta$ is also surjective and the result follows.

4.2. The following is well known; see [V, I, Proposition 7] for example.

Proposition. Let $R$ be a $k$-algebra which has a filtration $k \subseteq R_{0} \subseteq R_{1} \subseteq \cdots \subseteq$ $R_{n} \subseteq \cdots \subseteq \cup_{n \geqslant 0} R_{n}=R$ such that the associated graded ring $\mathrm{Gr} R=$ $\oplus_{n \geqslant 0} R_{n} / R_{n-1}$ is a finitely generated commutative $k$-algebra. Then

(i) $R$ is (left and right) Noetherian.

(ii) $R$ is finitely generated as a k-algebra.

Theorem A now follows immediately from Theorem 4.1 and Proposition 4.2.

4.3. We next show that the kernel of $\operatorname{Gr} \theta$ is $\Pi_{1}(\operatorname{Ker} \theta)$. For this we need the following lemma.

LEMMA. Let $\psi: \mathbf{Q}^{s} \rightarrow \mathbf{Q}^{n}$ be an epimorphism of vector spaces, and extend $\psi$ to a map $k\left[X_{1}, \ldots, X_{s}\right] \rightarrow k\left[x_{1}, \ldots, x_{n}\right]$, also denoted by $\psi$, where $X_{1}, \ldots, X_{s}$ and $x_{1}, \ldots, x_{n}$ are bases for $\mathbf{Q}^{s}$ and $\mathbf{Q}^{n}$ respectively. If $g=g\left(X_{1}, \ldots, X_{s}\right)$ is a homogeneous polynomial of degree $t$, and $\operatorname{deg} \psi g<t$, then $\psi g=0$.

Proof. We can by renumbering assume that $\psi X_{1}, \ldots, \psi X_{n}$ form a basis for $\mathbf{Q}^{n}$. Then for $n+1 \leqslant i \leqslant s$ we have

$$
X_{i}-\sum_{j=1}^{n} a_{i j} X_{j} \in \operatorname{Ker} \psi \quad \text { for some } a_{i j} \in k .
$$

Hence we may write

$$
\begin{aligned}
g\left(X_{1}, \ldots, X_{s}\right) & =g\left(X_{1}, \ldots, X_{n}, \sum_{j=1}^{n} a_{n+1} X_{j}, \ldots, \sum_{j=1}^{n} a_{s j} X_{j}\right)+g_{2}\left(X_{1}, \ldots, X_{s}\right) \\
& =g_{1}\left(X_{1}, \ldots, X_{n}\right)+g_{2}\left(X_{1}, \ldots, X_{s}\right)
\end{aligned}
$$


where $g_{2} \in \operatorname{ker} \psi$ and $g_{1}$ is either zero or a homogeneous polynomial of degree $t$ in $X_{1}, \ldots, X_{n}$. The result follows since the restriction of $\psi$ to $k\left[X_{1}, \ldots, X_{n}\right]$ is an isomorphism which preserves degrees.

\subsection{TheOREM. $\operatorname{Ker} \operatorname{Gr} \theta=\Pi_{1}(\operatorname{Ker} \theta)$.}

Proof. It is clear that $\Pi_{1}(\operatorname{Ker} \theta) \subseteq \operatorname{Ker} \operatorname{Gr} \theta$. Suppose $y \in \operatorname{Ker} \operatorname{Gr} \theta$. We may assume that $y=\Pi_{1}(x)$, where $x=\sum_{\lambda \in \mathbf{Z}^{s} \cap \nu} Q^{\lambda} g_{\lambda} \in D(Y)^{G}$ and each $g_{\lambda}$ is a homogeneous polynomial of degree $t$ in $Q_{1} P_{1}, \ldots, Q_{s} P_{s}$. Then $\theta\left(Q^{\lambda} g_{\lambda}\right)=0$ for $\lambda \notin \mathbf{Z} \Lambda$, so $\boldsymbol{\theta}\left(\sum_{\lambda \in \mathbf{Z}^{s} \cap \nu} Q^{\lambda} g_{\lambda}\right)=\sum_{\lambda \in \mathbf{Z} \Lambda} q^{\phi(\lambda)} \theta\left(g_{\lambda}\right)$. Since $y \in \operatorname{Ker} \operatorname{Gr} \theta$ we have $\theta(x) \in D_{t-1}$, so each polynomial $\theta\left(g_{\lambda}\right)$ has degree $\leqslant t-1$ in the variables $q_{1} p_{1}, \ldots, q_{n} p_{n}$. By the proof of Theorem $\mathrm{B}$, the restriction of $\theta$ to a map from $A$ to $B$ is a map of the type described in Lemma 4.3. It follows that $\theta\left(g_{\lambda}\right)=0$ for each $\lambda$ and so $x \in \operatorname{Ker} \theta$ and $y \in \Pi_{1}(\operatorname{Ker} \theta)$.

4.5. We draw a further consequence from this. Recall that a sequence of elements $x_{1}, \ldots, x_{r}$ in a commutative ring $R$ is an $R$-sequence provided $\sum_{i=1}^{r} x_{i} R \neq R$ and for each $i=1, \ldots, r$ the image of $x_{i}$ in $R / \sum_{j=1}^{i-1} x_{j} R$ is a nonzero divisor (see [K, 3.1]).

Lemma. Assume $G$ satisfies the dimension equality on $\mathcal{O}\left(U_{\beta}\right)$ for $\beta \in \Delta$. Then $\operatorname{Ker} \operatorname{Gr} \theta$ is generated by an $R$-sequence.

Proof. We can write $A=k\left[m_{1}, \ldots, m_{r}, h_{1}, \ldots, h_{t}\right]$ where $m_{1}, \ldots, m_{r}$ generate the ideal of $A$ consisting of functions which vanish on $\mathbf{Q} \Lambda$. Then $D\left(U_{\mathbf{s}}\right)^{G}=$ $\oplus_{\lambda \in \mathbf{z} \Lambda} D\left(U_{\mathbf{s}}\right)^{G}(\lambda)$ where $D\left(U_{\mathbf{s}}\right)^{G}(\lambda)=Q^{\lambda} A$. Let $X^{\lambda}=\Pi_{1}\left(Q^{\lambda}\right), Z_{i}=\Pi_{1}\left(m_{i}\right)$ for $1 \leqslant i \leqslant r$, and $Z_{i}=\Pi_{1}\left(h_{i-r}\right)$ for $r+1 \leqslant i \leqslant r+t=s$. Then

$$
\begin{aligned}
\operatorname{Gr} D\left(U_{\mathbf{s}}\right)^{G} & =k\left[X^{\lambda}, Z_{i}, \lambda \in \mathbf{Z} \Lambda, 1 \leqslant i \leqslant s\right] \\
& =k\left[X^{\lambda}\right]\left[Z_{1}, \ldots, Z_{s}\right] \text { a polynomial ring over } k\left[X^{\lambda}\right] .
\end{aligned}
$$

Now $D(Y)^{G}$ is a subring of $D\left(U_{\mathrm{s}}\right)^{G}$ and we have

$$
\operatorname{Gr} D(Y)^{G}=\bigoplus_{\lambda \in \mathbf{Z} \Lambda} X^{\lambda} \Pi_{1}\left(S_{\lambda}\right) k\left[Z_{1}, \ldots, Z_{s}\right] \text {. }
$$

By Theorem 4.4 and Corollary 3.4, $\operatorname{Ker} \operatorname{Gr} \theta$ is the ideal of $\operatorname{Gr} D(Y)^{G}$ generated by $Z_{1}, \ldots, Z_{r}$. By the proof of Lemma 3.5 we have

$$
\Pi_{1}\left(S_{\lambda}\right) k\left[Z_{1}, \ldots, Z_{s}\right] \cap \sum_{i=1}^{l} Z_{i} k\left[Z_{1}, \ldots, Z_{s}\right]=\Pi_{1}\left(S_{\lambda}\right) \sum_{i=1}^{l} Z_{i} k\left[Z_{1}, \ldots, Z_{s}\right]
$$

for $1 \leqslant l \leqslant r$ and hence

$$
\sum_{i=1}^{l} \operatorname{Gr} D\left(U_{\mathbf{s}}\right)^{G} Z_{i} \cap \operatorname{Gr} D(Y)^{G}=\sum_{i=1}^{l} \operatorname{Gr} D(Y)^{G} Z_{i}
$$

Since the elements $Z_{1}, \ldots, Z_{r}$ form an $R$-sequence in the ring $\operatorname{Gr} D\left(U_{\mathrm{s}}\right)^{G}$ it follows from the equality above that they form an $R$-sequence in $\operatorname{Gr} D(Y)^{G}$.

4.6. Our next aim is to show that the graded rings $\operatorname{Gr} D(Y)^{G}$ and $\operatorname{Gr} D\left(Y_{\Delta} / G\right)$ are Gorenstein rings. We have

$$
\operatorname{Gr} D(Y)=k\left[X_{1}, \ldots, X_{s}, Y_{1}, \ldots, Y_{s}, X_{i}^{-1} ; i \in \bigcap_{\beta \in \Delta} \beta\right]
$$


and $G$ acts on $V=\operatorname{span}\left\{X_{1}, \ldots, X_{s}, Y_{1}, \ldots, Y_{s}\right\}$ as a subgroup of $\operatorname{SL}(V)$. Hence the fact that $D(Y)^{G}$ is Gorenstein follows from the following which is a slight extension of a result of R. P. Stanley [S, p. 81].

TheOREM. Assume the torus $G$ acts as a diagonal subgroup of $\operatorname{SL}(V)$ where $V=k^{s}$. For a fixed subset $\beta$ of $\{1,2, \ldots, s\}$ let $U=\left\{v \in V \mid v_{i} \neq 0\right.$ if $\left.i \in \beta\right\}$. Then $\mathcal{O}(U)^{G}$ is a Gorenstein ring.

We can assume that $\mathcal{O}(U)=k\left[T_{1}^{ \pm 1}, \ldots, T_{t}^{ \pm 1}, T_{t+1}, \ldots, T_{s}\right]$, and that as explained in $\S 1, \mathcal{O}(U)^{G}$ is a semigroup algebra $k \Lambda$ where $\Lambda$ has the form

$$
\Lambda=\left\{b \in \mathbf{Z}^{t} \times\left(\mathbf{Z}_{+}\right)^{s-t} \mid f_{i}(b)=0 \text { for } 1 \leqslant i \leqslant r\right\}
$$

for certain linear equations $f_{i}\left(x_{1}, \ldots, x_{s}\right)=\sum_{j=1}^{s} a_{i j} x_{j} \in\left(\mathbf{Q}^{s}\right)^{*}$.

A subsemigroup $M$ of $\mathbf{Z}_{+}^{s}$ will be called a positive toroidal semigroup if it is the set of solutions in nonnegative integers to a system of homogeneous linear equations with integer coefficients and there exists an element $m=\left(m_{1}, \ldots, m_{s}\right) \in M$ with $m_{i}>0$ for each $i$. If $M$ is a positive toroidal semigroup, an element $m=$ $\left(m_{1}, \ldots, m_{s}\right) \in M$ is a minimal positive element provided $m_{i}>0$ for each $i$, and whenever $n=\left(n_{1}, \ldots, n_{s}\right) \in M$ with $n_{i}>0$ for each $i$, we have $m_{i} \leqslant n_{i}$. Theorem 4.6 is deduced from the following result of Stanley.

4.7. THEOREM. If $M$ is a positive toroidal semigroup, then $k M$ is a Gorenstein ring if and only if $M$ has a unique minimal positive element.

Proof. [S, Theorem 6.7].

4.8. Lemma. If $\mathcal{O}(U)^{G}=k \Lambda$ where $G \subseteq \operatorname{SL}(V)$ as in Theorem 4.6 then $k \Lambda=$ $(k M)_{\mathscr{C}}$ is a localization of a semigroup algebra $k M$, where $M$ is isomorphic to a positive toroidal semigroup with a unique minimal positive element.

Together with Theorem 4.7, and the fact that a localization of a Gorenstein ring is again a Gorenstein ring [K, Exercise 12, p. 164], this will prove Theorem 4.6.

Proof of Lemma 4.8. The hypothesis $G \subseteq \operatorname{SL}(V)$ is equivalent to the fact that $\sum_{j=1}^{s} a_{i j}=0$ for $1 \leqslant i \leqslant r$. That is $f_{1}, \ldots, f_{r}$ belong to the subspace $W$ of $\left(\mathbf{Q}^{s}\right)^{*}$ spanned by $x_{1}-x_{2}, x_{2}-x_{3}, \ldots, x_{s-1}-x_{s}$. Let $\left(b_{i j}\right)$ be the row reduced form of the matrix $\left(a_{i j}\right)$ and $g_{i}(x)=\sum b_{i j} x_{j}$. Then $g_{i} \in W$ for each $i$, and $\Lambda$ is the set of solutions in $\mathbf{Z}^{t} \times\left(\mathbf{Z}_{+}\right)^{s-t}$ to the equations $g_{i}(x)=0$.

As usual the variables $x_{j}$ corresponding to a column of $\left(b_{i j}\right)$ containing a leading one are called leading variables, and the others are nonleading variables. We can assign the nonleading variables arbitrary values and solve for the leading variables. Moreover the value of a leading variable $x_{j}$ is determined by the values of the nonleading variables $x_{k}$ with $k \geqslant j$. Therefore we can assign a value zero to every variable $x_{j}$ with $j \geqslant t+1$ and assign the remaining nonleading variables arbitrary values.

For each nonleading variable $x_{j}$ with $1 \leqslant j \leqslant t$, we obtain in this way a solution $\beta_{j}=\left(\beta_{j 1}, \ldots, \beta_{j s}\right) \in \Lambda$ with $\beta_{j j}$ a positive integer and $\beta_{j k}=0$ if $k \geqslant t+1$ or $x_{k}$ is any nonleading variable with $k \neq j$. 
Let $M$ be the subsemigroup of $\Lambda$ given by

$$
\begin{aligned}
M=\left\{b=\in \mathbf{Z}^{t} \times\left(\mathbf{Z}_{+}\right)^{s-t}\right. & \mid f_{i}(b)=0,1 \leqslant i \leqslant r \text { and } b_{j} \geqslant 0 \\
& \text { if } \left.x_{j} \text { is a nonleading variable with } 1 \leqslant j \leqslant t\right\}
\end{aligned}
$$

and let $\mathscr{C}$ be the multiplicatively closed subset of $k M$ generated by the set $\left\{T^{\beta_{j}}\right\}$. Then it follows easily that $k \Lambda=(k M)_{\mathscr{C}}$.

It remains to show that $M$ has the properties claimed. By changing notation, we may assume that

$$
\begin{aligned}
M=\left\{b \in \mathbf{Z}^{p} \times\left(\mathbf{Z}_{+}\right)^{s-p} \mid b_{i}=f_{i}\left(b_{p+1}, \ldots, b_{s}\right) \text { for } 1 \leqslant i \leqslant p\right. \\
\left.\quad \text { and } g_{j}\left(b_{p+1}, \ldots, b_{s}\right)=0, j \in J\right\}
\end{aligned}
$$

where as before $x_{i}-f_{i}\left(x_{p+1}, \ldots, x_{s}\right)$ and $g_{j}\left(x_{p+1}, \ldots, x_{s}\right)$ belong to $W$. To see this first regroup all the variables which are constrained to be nonnegative at the end, and relabel them as $x_{p+1}, \ldots, x_{s}$. Then relabel the leading variables $x_{j}$ with $1 \leqslant j \leqslant t$ as $x_{1}, \ldots, x_{p}$ and express their dependence on $x_{p+1}, \ldots, x_{s}$ by the equations $x_{i}=f_{i}\left(x_{p+1}, \ldots, x_{s}\right)$. Any further relations between the $x_{p+1}, \ldots, x_{s}$ are expressed by certain equations $g_{j}\left(x_{p+1}, \ldots, x_{s}\right)=0$, where $j$ runs over some index set $J$.

Suppose $f_{i}\left(x_{p+1}, \ldots, x_{s}\right)^{\prime}=\sum_{k=p+1}^{s} c_{i k} x_{k}$ where $c_{i k} \in \mathbf{Q}$ and choose $m \in \mathbf{Z}$ such that $m+c_{i k}>0$ for each $i$ and $k$. For $1 \leqslant i \leqslant p$ define $h_{i}(x)=x_{i}+m \sum_{k=p+1}^{s} x_{k}$ and let $\zeta: \mathbf{Q}^{s} \rightarrow \mathbf{Q}^{s}$ be the linear map defined by

$$
\zeta\left(b_{1}, \ldots, b_{s}\right)=\left(h_{1}(b), \ldots, h_{p}(b), b_{p+1}, \ldots, b_{s}\right) .
$$

It is easily checked that $\zeta$ is an isomorphism and that

$$
\begin{aligned}
\zeta(M)=\left\{\left(c_{1}, \ldots, c_{s}\right) \in \mathbf{Z}_{+}^{s} \mid c_{i}=f_{i}\left(c_{p+1}, \ldots, c_{s}\right)+m \sum_{k=p+1}^{s} c_{k}\right. \\
\left.\quad \text { for } 1 \leqslant i \leqslant p \text { and } g_{j}\left(c_{p+1}, \ldots, c_{s}\right)=0, j \in J\right\} .
\end{aligned}
$$

Hence $M$ is isomorphic to the positive toroidal semigroup $\zeta(M)$. For $1 \leqslant i \leqslant p$, let $a_{i}=f_{i}(1,1, \ldots, 1)+m(s-p)$. Then since $g_{j} \in W$ it follows that $\left(a_{1}, \ldots, a_{p}, 1,1, \ldots, 1\right)$ is the unique minimal positive element of $\zeta(M)$. This completes the proof.

TheOREM D. The graded rings $\operatorname{Gr} D(Y)^{G}$ and $\operatorname{Gr} D\left(Y_{\Delta} / G\right)$ are Gorenstein rings.

Proof. The fact that $\operatorname{Gr} D(Y)^{G}$ is Gorenstein now follows directly from Theorem 4.6. To prove $\operatorname{Gr} D\left(Y_{\Delta} / G\right)$ is Gorenstein we may assume by Proposition 3.7 that $G$ satisfies the dimension equaltiy on $\mathcal{O}\left(U_{\beta}\right)$ for $\beta \in \Delta$, and the map $\theta: D(Y)^{G} \rightarrow$ $D\left(Y_{\Delta} / G\right)$ is surjective. Hence the map $\operatorname{Gr} \theta: \operatorname{Gr} D(Y)^{G} \rightarrow \operatorname{Gr} D\left(Y_{\Delta} / G\right)$ is surjective, and by Lemma 4.5 the kernel of $\operatorname{Gr} \theta$ is generated by an $R$-sequence. It follows that $\operatorname{Gr} D\left(Y_{\Delta} / G\right)$ is Gorenstein by [K, Exercise 13, p. 164]. 
4.9. The fact that these graded rings are Gorenstein can be used to apply a result of Levasseur, which we explain next.

Let $A$ be a filtered ring such that the associated graded ring $B$ is commutative Noetherian with $\operatorname{Kd}(B)=\omega$, where $\mathrm{Kd}$ denotes Krull dimension. If $M$ is a finitely generated $A$-module, we may filter $M$ in such a way that the associated graded module $\operatorname{Gr}(M)$ is a finitely generated $B=\operatorname{Gr}(A)$-module, and we set $d(M)=$ $\operatorname{Kd}\left({ }_{B} \operatorname{Gr}(M)\right)$. We set $\operatorname{grade}(M)=\inf \left\{i \mid \operatorname{Ext}_{A}^{i}(M, A) \neq 0\right\}$. We say that $B$ is of pure dimension $\omega$ if for every maximal ideal $m$ of $B, \operatorname{Kd}\left(B_{m}\right)=\omega$. The next result is [L2, Théorème 4.4].

THEOREM. Suppose that $B$ is a Gorenstein ring of pure dimension $\omega$. Then for any finitely generated, nonzero $A$-module $M$, we have $d(M)+\operatorname{grade}(M)=\omega$.

In addition the left and right injective dimensions of $A$ are finite and equal to $\mu \leqslant \omega$.

4.10. We note that if $B$ is a finitely generated domain over a field $k$, and $\operatorname{Kd}(B)=\omega$, then $B$ has pure dimension $\omega$. This follows from Noether's normalization lemma and the going up and going down theorems.

It is therefore of interest to compute the Krull dimensions of the graded rings of Theorem D. More generally in the next lemma we assume that $R$ is a subring of $D\left(U_{\mathrm{s}}\right)=k\left[Q_{1}^{ \pm 1}, \ldots, Q_{s}^{ \pm 1}, P_{1}, \ldots, P_{s}\right]$ containing $A=k\left[Q_{1} P_{1}, \ldots, Q_{s} P_{s}\right]$. By Lemma 2.2, $R=\bigoplus_{\lambda \in \mathbf{Z}^{s}} R(\lambda)$ where $R(\lambda)=\left\{x \in R \|\left[Q_{i}, P_{i}, x\right]=\lambda_{i} x\right\}$. Let $\Lambda=\{\lambda \in$ $\left.\mathbf{Z}^{s} \mid R(\lambda) \neq 0\right\}$, and suppose $\operatorname{rank}(\mathbf{Z} \Lambda)=r$.

Let $\mathscr{E}_{t}$ be the span of all differential operators of order at most $t$ in $D\left(U_{\mathrm{s}}\right)$ and $\operatorname{Gr}\left(D\left(U_{\mathrm{s}}\right)\right)=\oplus \mathscr{E}_{t} / \mathscr{E}_{t-1}$. If $x \in \mathscr{E}_{t}-\mathscr{E}_{t-1}$ we define $\Pi(x)=\left(x+\mathscr{E}_{t}\right) / \mathscr{E}_{t-1}$. Then $\operatorname{Gr}(R)=\Pi(R)$ is a subring of $\operatorname{Gr}\left(D\left(U_{\mathrm{s}}\right)\right)$.

If $\operatorname{Gr}(R)$ is a finitely generated $k$-algebra, $\operatorname{Kd}(\operatorname{Gr}(R))=\operatorname{tr} \operatorname{deg}_{k}(\operatorname{Gr}(R))$, the transcendence degree of $\operatorname{Gr}(R)$ over $k$. We compute this next.

LEMMA. With the above notation $\operatorname{tr} \operatorname{deg}_{k}(\operatorname{Gr}(R))=s+r$.

Proof. Let $S=k\left[Q_{1} P_{1}, \ldots, Q_{n} P_{n}, Q^{\lambda}, \lambda \in \mathbf{Z} \Lambda\right]$. Then $R \subset S, \operatorname{Gr}(R) \subset \operatorname{Gr}(S)$, and $\operatorname{tr} \operatorname{deg}_{k}(\operatorname{Gr}(S))=s+r$. It suffices to show that $\operatorname{Gr}(R)$ and $\operatorname{Gr}(S)$ have the same quotient fields, and this is proved in the same way as Lemma 3.3.

4.11. Corollary. (i) Suppose $G=\mathrm{GL}(1)^{r}$ acts faithfully on $k^{s}$. Then $\operatorname{Kd~Gr} D(Y)^{G}=2 s-r$.

(ii) Let $Y=\cup U_{\beta}$ and suppose the common quotient field of the rings $\mathcal{O}\left(U_{\beta}\right)^{G}$ has transcendence degree $n$ over $k$. Then $\operatorname{Kd~} \mathrm{Gr} D\left(Y_{\Delta} / G\right)=2 n$.

Proof. Since $\operatorname{Gr} D(Y)^{G}$ and $\operatorname{Gr} D\left(Y_{\Delta} / G\right)$ are finitely generated $k$-algebras by Theorem 4.1, this follows from Lemma 4.10 and Theorems 2.4 and 2.7.

\section{REFERENCES}

[BB] W. Borho and J.-L. Brylinski, Differential operators on homogeneous spaces. I, Invent. Math. 69 (1982), 437-476.

[B] A. Brondsted, An introduction to convex polytopes, Graduate Texts in Math., no. 90, Springer-Verlag, Berlin, Heidelberg and New York, 1982.

[H] R. Hart, Differential operators on affine algebras, J. London Math. Soc. (2) 28 (1983), 470-476. 
[Ho] M. Hochster, Rings of invariants of tori, Cohen-Macaulay rings generated by monomials, and polytopes, Ann. of Math. (2) 96 (1972), 318-337.

[Hu] J. E. Humphreys, Linear algebraic groups, Graduate Texts in Math., no. 21, Springer-Verlag, Berlin, Heidelberg, and New York, 1975.

[Ka] J. M. Kantor, Formes et opérateurs différentiels sur les espaces analytiques complexes, Bull. Soc. Math. France 53 (1977), 5-80.

[K] I. Kaplansky, Commutative rings, Univ. of Chicago Press, Chicago and London, 1974.

[Ke] G. Kempf et al., Toroidal embeddings. I, Lecture Notes in Math., no. 339, Springer-Verlag, Berlin, Heidelberg, and New York, 1973.

[KL] G. R. Krause and T. H. Lenagan, Growth of algebras and Gelfand-Kirillov dimension, Research Notes in Math., No. 116, Pitman, Boston.

[L1] T. Levasseur, Anneaux d'opérateurs différentiels, Séminaire d'Algèbre Paul Dubreil et Marie-Paule Malliavin, Lecture Notes in Math., no. 867, Springer-Verlag, Berlin, Heidelberg, and New York, 1981, pp. $157-173$.

[L2] Complexe bidualisant en algèbre non-commutative, Séminaire d'Algèbre Paul Dubreil et Marie-Paule Malliavin, Lecture Notes in Math., no. 1146, Springer-Verlag. Berlin, Heidelberg, and New York, 1985, pp. 397-415.

[M] I. M. Musson, Actions of tori on Weyl algebras, Comm. Algebra (to appear).

[S] R. P. Stanley, Hilbert functions of graded algebras, Adv. in Math. 28 (1978), 57-83.

[V] J. P. Vigué, Opérateurs différentiels sur les espaces analytiques, Invent. Math. 20 (1973), 313-336.

Department of Mathematics, University of Wisconsin-Milwaukee, Milwaukee, Wisconsin 53201 